\title{
Deep Learning for Neuroimaging-based Diagnosis and Rehabilitation of Autism Spectrum Disorder: A Review
}

\author{
Marjane Khodatars, Afshin Shoeibi, Delaram Sadeghi, Navid Ghassemi, Mahboobeh Jafari, Parisa Moridian, \\ Ali Khadem, Roohallah Alizadehsani, Assef Zare, Yinan Kong, Abbas Khosravi, Saeid Nahavandi, \\ Sadiq Hussain, U. Rajendra Acharya, Michael Berk
}

\begin{abstract}
Accurate diagnosis of Autism Spectrum Disorder (ASD) followed by effective rehabilitation is essential for the management of this disorder. Artificial intelligence (AI) techniques can aid physicians to apply automatic diagnosis and rehabilitation procedures. AI techniques comprise traditional machine learning (ML) approaches and deep learning (DL) techniques. Conventional ML methods employ various feature extraction and classification techniques, but in DL, the process of feature extraction and classification is accomplished intelligently and integrally. DL methods for diagnosis of ASD have been focused on neuroimaging-based approaches. Neuroimaging techniques are non-invasive disease markers potentially useful for ASD diagnosis. Structural and functional neuroimaging techniques provide physicians substantial information about the structure (anatomy and structural connectivity) and function (activity and functional connectivity) of the brain. Due to the intricate structure and function of the brain, proposing optimum procedures for ASD diagnosis with neuroimaging data without exploiting powerful AI techniques like DL may be challenging. In this paper, studies conducted with the aid of DL networks to distinguish ASD are investigated. Rehabilitation tools provided for supporting ASD patients utilizing DL networks are also assessed. Finally, we will
\end{abstract}

M. Khodatars and D. Sadeghi are with the Dept. of Medical Engineering, Mashhad Branch, Islamic Azad University, Mashhad, Iran.

A. Shoeibi and N. Ghassemi are with the Faculty of Electrical Engineering, FPGA Lab, K. N. Toosi University of Technology, Tehran, Iran, and the Computer Engineering Department, Ferdowsi University of Mashhad, Mashhad, Iran. (Corresponding author: Afshin Shoeibi, email: afshin.shoeibi@gmail.com).

M. Jafari is with Electrical and Computer Engineering Faculty, Semnan University, Semnan, Iran.

P. Moridian is with the Faculty of Engineering, Science and Research Branch, Islamic Azad University, Tehran, Iran.

A. Khadem is with the Faculty of Electrical Engineering, K. N. Toosi University of Technology, Tehran, Iran. (Corresponding author: Ali Khadem, email: alikhadem@kntu.ac.ir).

R. Alizadehsani, A. Khosravi and S. Nahavandi. are with the Institute for Intelligent Systems Research and Innovation (IISRI), Deakin University, Victoria 3217, Australia.

A. Zare is with Faculty of Electrical Engineering, Gonabad Branch, Islamic Azad University, Gonabad, Iran.

Y. Kong is with the School of Engineering, Macquarie University, Sydney, 2109, Australia.

S. Hussain is with the Dibrugarh University, Assam, India, 786004.

U. R. Acharya is with the Dept. of Electronics and Computer Engineering, Ngee Ann Polytechnic, Singapore 599489, Singapore, the Dept. of Biomedical Informatics and Medical Engineering, Asia University, Taichung, Taiwan, and the Dept. of Biomedical Engineering, School of Science and Technology, Singapore University of Social Sciences, Singapore.

M. Berk is with the Deakin University, IMPACT - the Institute for Mental and Physical Health and Clinical Translation, School of Medicine, Barwon Health, Geelong, Australia, and the Orygen, The National Centre of Excellence in Youth Mental Health, Centre for Youth Mental Health, Florey Institute for Neuroscience and Mental Health and the Department of Psychiatry, The University of Melbourne, Melbourne, Australia. present important challenges in the automated detection and rehabilitation of ASD and propose some future works.

Index Terms-Autism Spectrum Disorder, Diagnosis, Rehabilitation, Deep Learning, Neuroimaging, Neuroscience.

\section{INTRODUCTION}

A $\mathrm{SD}$ is a disorder of the nervous system that affects the brain and results in difficulties in speech, social interaction and communication deficits, repetitive behaviors, and delays in motor abilities [1]. This disease can generally be distinguished with extant diagnostic protocols from the age of three years onwards. Autism influences many parts of the brain. This disorder also involves a genetic influence via the gene interactions or polymorphisms [2], [3]. One in 70 children worldwide is affected by autism. In 2018, the prevalence of ASD was estimated to occur in 168 out of 10,000 children in the United States, one of the highest prevalence rates worldwide. Autism is significantly more common in boys than in girls. In the United States, about 3.63 percent of boys aged 3 to 17 years have autism spectrum disorder, compared with approximately 1.25 percent of girls [4].

Diagnosing ASD is difficult because there is no pathophysiological marker, relying instead just on psychological criteria [5]. Psychological tools can identify individual behaviors, levels of social interaction, and consequently facilitate early diagnosis. Behavioral evaluations embrace various instruments and questionnaires to assist the physicians to specify the particular type of delay in a child's development, including clinical observations, medical history, autism diagnosis instructions, and growth and intelligence tests [6].

Several investigations for the diagnosis of ASD have recently been conducted on neuroimaging data (structural and functional).

Analyzing anatomy and structural connections of brain areas with structural neuroimaging is an essential tool for studying structural disorders of the brain in ASD. The principal tools for structural brain imaging are magnetic resonance imaging (MRI) techniques [7], [8], [9]. Cerebral anatomy is investigated by structrul MRI (sMRI) images and anatomical connections are assesed by diffusion tensor imaging MRI (DTI-MR) [10]. Investigating the activity and functional connections of brain areas using functional neuroimaging can also be used for studying ASD. Brain functional diagnostic 
tools are older approaches than structural methods for studying ASD. The most basic modality of functional neuroimaging is electroencephalography (EEG), which records the electrical activity of the brain from the scalp with a high temporal resolution (in milliseconds order) [11]. Studies have shown that employing EEG signals to diagnose ASD have been useful [12], [13], [14]. Functional MRI (fMRI) is one of the most promising imaging modalities in functional brain disorders, used as task-based (T-fMRI) or resting-state (rs-fMRI) [15], [16]. fMRI-based techniques have a high spatial resolution (in the order of millimeters) but a low temporal resolution due to slow response of the hemodynamic system of the brain as well as fMRI imaging time constraints and is not ideal for recording the fast dynamics of brain activities. In addition, these techniques have a high sensitivity to motion artifacts. It should be stressed that in consonance with studies, three less prevalent modalities of electrocorticography (ECoG) [17], functional near-infrared spectroscopy (fNIRS) [18], and Magnetoencephalography (MEG) [19] can also attain reasonable performance in ASD diagnosis. An appropriate approach is to utilize machine-learning techniques alongside functional and structural data to collaborate with physicians in the process of accurately assessing ASD. In the field of ASD, applying machine learning methods generally entail two categories of traditional methods [20] and DL methods [21]. As opposed to traditional methods, much less work has been done on DL methods to explore ASD or design rehabilitation tools.

This study reviews ASD assesment methods and patients' rehabilitation with DL networks. The outline of this paper is as follows. Section 2 is search strategy. Section 3 concisely presents the DL networks employed in the field of ASD. In section 4, existing computer-aided diagnosis systems (CADS) that use brain functional and structural data are reviewed. In section 5, DL-based rehabilitation tools for supporting ASD patients are introduced. Section 6 discusses the reviewed papers. Section 7 reveals the challenges of ASD diagnosis and rehabilitation with DL. Finally, the paper concludes and suggests future work in section 8 .

\section{SEARCh Strategy}

In this review, IEEE Xplore, ScienceDirect, SpringerLink, $\mathrm{ACM}$, as well as other conferences or journals were used to acquire papers on ASD diagnosis and rehabilitation using DL methods. Further, the keywords "ASD", "Autism Spectrum Disorder" and "Deep Learning" were used to select the papers. The papers are analyzed till June 03th, 2020 by the authors (AK, SN). Figure 1 depicts the number of considered papers using DL methods for the automated detection and rehabilitation of ASD each year.

\section{DEEP LEARNING TECHNIQUES FOR ASD DiAgNOSIS AND REHABILITATION}

Nowadays, DL algorithms are used in many areas of medicine including structural and functional neuroimaging. The application of DL in neural imaging ranges from brain MR image segmentation [22], to detection of brain lesions such as tumors [23], diagnosis of brain functional disorders

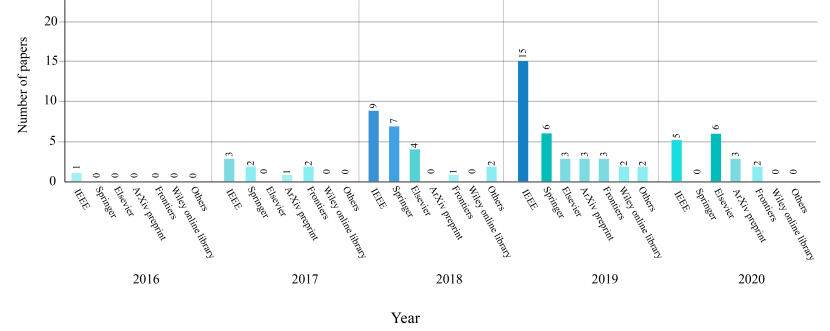

Fig. 1: Number of papers published every year for ASD diagnosis and rehabilitation.

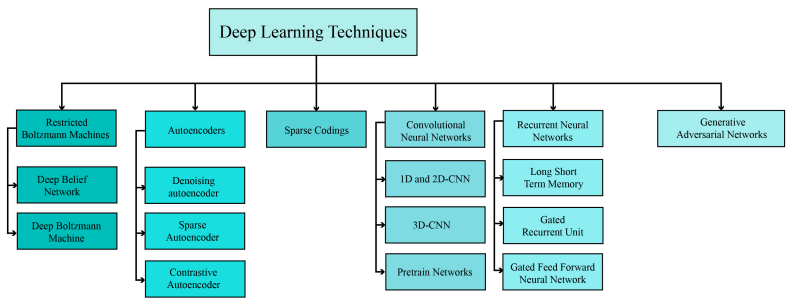

Fig. 2: Illustration of various types of DL methods.

such as ASD [24], and production of artificial structural or functional brain images [25]. Machine learning techniques are categorized into three fundamental categories of learning: supervised learning [26], unsupervised learning [27], and reinforcement learning [28], and a variety of DL networks are provided for each type. So far, most studies applied to identify ASD using DL have been based on supervised or unsupervised approaches. Figure 2 illustrates generally employed types of DL networks with supervised or unsupervised learning to study ASD.

\section{CADS-BASED DEEP LEARNING TECHNIQUES FOR ASD DIAGNOSIS BY NEUROIMAGING DATA}

A traditional artificial intelligence (AI)-based CADS encompasses several stages of data acquisition, data preprocessing, feature extraction, and classification [29], [30], [31], [32]. In [33], [34], [35] existing traditional algorithms for diagnosing ASD have been reviewed. In contrast to traditional methods, in DL-based CADS, feature extraction, and classification are performed intelligently within the model. Also, due to the structure of DL networks, using large dataset to train DL networks and recognize intricate patterns in datasets is incumbent. The components of DL-based CADS for ASD detection are shown in Figure 3 . It can be noted from the figure that, large and free databases are first introduced to diagnose ASD. In the second step, various types of preprocessing techniques are used on functional and structural data to be scrutinized. Finally, the DL networks are applied on the preprocessed data. 


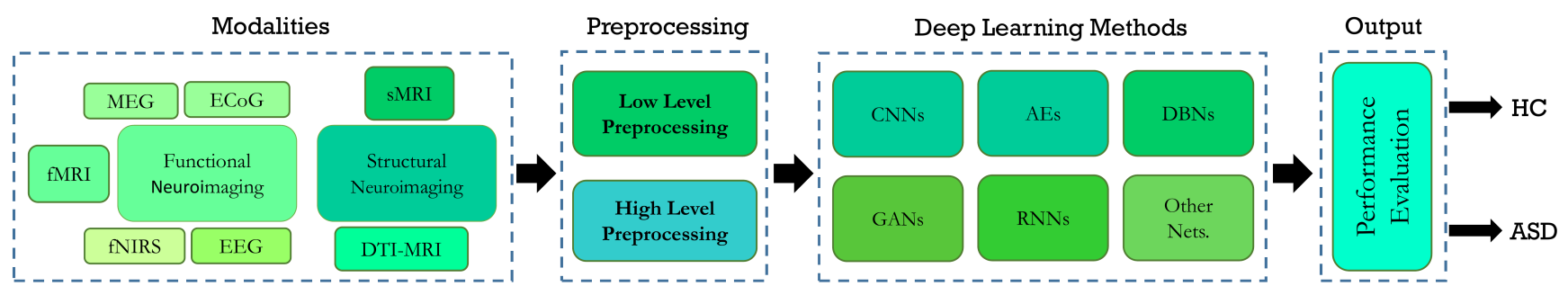

Fig. 3: Block diagram of CAD system using DL architecture for ASD detection.

\section{A. Neuroimaging ASD Datasets}

Datasets are the heart of any CADS development and the capability of CADS depends primarily on the affluence of the input data. To diagnose ASD, several brain functional and structural datasets are available. The most complete free dataset available is ABIDE [36] dataset with two subsets: ABIDE-I and ABIDE-II, which encompasses sMRI, rs-fMRI, and phenotypic data. ABIDE-I involves data from 17 international sites, yielding a total of 1112 datasets, including 539 individuals with ASD and 573 healthy individuals (ages 64-7). In accordance with HIPAA guidelines and 1000 FCP / INDI protocols, these data are anonymized. In contrast, ABIDEII contains data from 19 international sites, with a total of 1114 datasets from 521 individuals with ASD and 593 healthy individuals (ages 5-64). Also, preprocessed images of the ABIDE-I series called PCP [37] can be freely downloaded by the researchers. The second recently released ASD diagnostic database is called NDAR, which comprises various modalities, and more information is provided in [38].

\section{B. Preprocessing Techniques}

Neuroimaging data (especially functional ones) is of relatively complicated structure, and if not pre-processed properly, it may affect the final diagnosis. Preprocessing of this data typically entails multiple common steps performed by different software as standard. Indeed, occasionally prepared pipelines are applied on the dataset to yield pre-processed data for future researches. In the following section, preprocessing steps are briefly explained for fMRI data.

1) Standard (Low-level) fMRI preprocessing steps: Lowlevel pre-processing of fMRI images normally has fixed number of steps applied on the data, and prepared toolboxes are usually used to reduce execution time and yield better accuracy. Some of these reputable toolboxes contain FMRIB software libraries (FSL) [39], BET [40], FreeSurfer [41], and SPM [42]. Also, important and vital fMRI preprocessing incorporates brain extraction, spatial smoothing, temporal filtering, motion correction, slice timing correction, intensity normalization, and registration to standard atlas, which are summarized as follows:

BRAIN EXTRACTION: the goal is to remove the skull and cerebellum from the fMRI image and maintain the brain tissue [43], [44], [45].

SPATIAL SMOOTHING: involves averaging the adjacent voxels signal. This process is persuasive on account of neighbor- ing brain voxels being usually closely related in function and blood supply [43], [44], [45].

TEMPORAL FILTERING: the aim is to eliminate unwanted components from the time series of voxels without impairing the signal of interest [43], [44], [45].

REALIGNMENT (Motion CORRECTION): During the fMRI test, people often move their heads. The objective of motion correction is to align all images to a reference image so that the coordinates and orientation of the voxels be identical in all fMRI volumetric images [43], [44], [45].

SLICE TIMING CORRECTION: The purpose of modifying the slice time is to adjust the time series of the voxels so that all the voxels in each fMRI volume image have a common reference time. Usually, the corresponding time of the first slice recorded in each fMRI volume image is selected as the reference time [43], [44], [45].

INTENSITY NORMALIZATION: at this stage, the average intensity of fMRI signals are rescaled to compensate for global deviations within and between the recording sessions [43], [44], [45].

REGISTRATION TO A STANDARD ATLAS: The human brain entails hundreds of cortical and subcortical areas with various structures and functions, each of which is very timeconsuming and complex to study. To overcome the problem, brain atlases are employed to partition brain images into a confined number of ROIs, following which the mean time series of each ROI can be extracted [46]. ABIDE datasets use a manifold of atlases, including Automated Anatomical Labeling (AAL) [47], Eickhoff-Zilles (EZ) [48], Harvard-Oxford (HO) [49], Talaraich and Tournoux (TT) [50], Dosenbach 160 [51], Craddock 200 (CC200) [52] and Craddock 400 (CC400) [53] and more information is provided in [54]. Table I provides complete information on preprocessing tools, atlases, and some other preprocessing information.

2) Pipeline Methods: Pipelines present preprocessed images of ABIDE databases. They embrace generic preprocessing procedures. Employing pipelines, distinct methods can be compared with each other. In ABIDE datasets, preprocessing is performed by four pipeline techniques: neuroimaging analysis kit (NIAK) [55], data processing assistant for rs- fMRI (DPARSF) [56], the configurable pipeline for the analysis of connectomes (CPAC) [57], or connectome computation system (CCS) [58]. The preprocessing steps carried out by the various pipelines are comparatively analogous. The chief differences are in the particular algorithms for each step, the software simulations, and the parameters applied. Details of each pipeline technique are provided in [54]. Table [] 


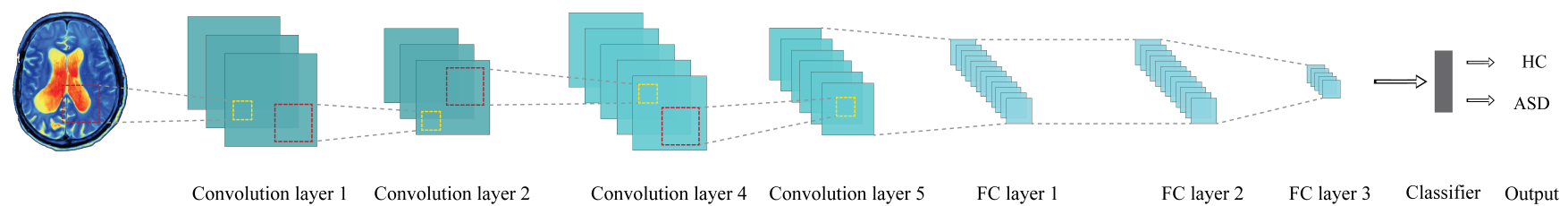

Fig. 4: Overall block diagram of a 2D-CNN used for ASD detection.

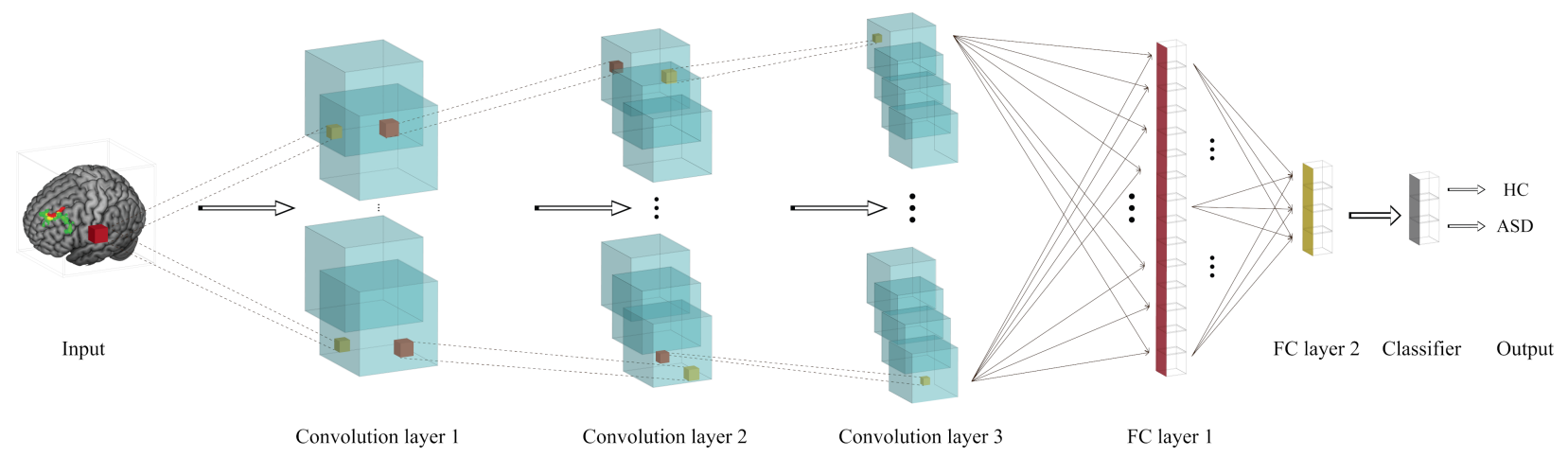

Fig. 5: Overall block diagram of a 3D-CNN used for ASD detection.

demonstrates the pipeline techniques used in autism detection exploiting DL.

3) High-level preprocessing Steps: High-level techniques for pre-processing brain data are important, and using them accompanying preliminary pre-processing methods can enhance the accuracy of ASD recognition. These methods are applied after the standard pre-processing of functional and structural brain data. These include sliding window (SW) [24], data augmentation (DA) [59], functional connectivity matrix (FCM) estimation [60], [61] and applying fast Fourier transformation (FFT) [62]. Furthermore, some of the researches utilized feature extraction [63] techniques and some also use feature selection methods. Precise information on reviewed studies is indicated in detail in Table I]

\section{Deep Neural Networks}

Deep learning in various medical applications, including the diagnosis of ASD, has become extremely popular in recent years. In this section of the paper, the types of Deep Learning networks used in ASD detection are examined, which include CNN, RNN, AE, DBN, CNN-RNN, and CNN-AE models.

1) Convolutional Neural Networks (CNNs) : In this section, the types of popular convolutional networks used in ASD diagnosis are surveyed. These networks involve 1D-CNN, 2DCNN, 3D-CNN models, and a variety of pre-trained networks such as VGG.

\section{D AND 2D-CNN}

There are many spatial dependancies present in the data and it is difficult to extract these hidden signatures from the data. Convolution network uses a structure alike to convolution filters to extract these features properly and contribute to the knowledge that features should be processed taking into account spatial dependencies; so the number of network parameters are significantly reduced. The principal application of these networks is in image processing and due to the two-dimensional (2D) image inputs, convolution layers form 2D structures, which is why these networks are called 2D convolutional neural network (2D-CNN). By using another type of data, one-dimensional signals, the convolution layers' structure also resembles the data structure [64]. In convolution networks, assuming that various data sections do not require learning different filters, the number of parameters are markedly lessened and make it feasible to train these networks with smaller databases [21]. Figure 4 shows the block digram of 2D-CNN used for ASD detection.

\section{D-CNN}

By transforming the data into three dimensions, the convolution network will also be altered to a three-dimensional format (Figure 5). It should be noted that the manipulation of three dimensional CNN (3D-CNN) networks is less beneficial than 1D-CNN and 2D-CNN networks for diverse reasons. First, the data required to train these networks must be much larger which conventionally such datasets are not utilizable and methods such as pre-training, which are extensively exploited in 2D networks, cannot be used here. Another reason is that with more complicated structure of networks, it becomes much tougher to fix the number of layers, and network structure. The 3D activation map generated during the convolution of a 3D CNN is essential for analyzing data where volumetric or temporal context is crucial. This ability to analyze a series of frames or images in context has led to the use of 3D CNNs as tools for action detection and evaluation of medical imaging. [65].

2) Deep Belief Networks (DBNs): DBNs are not popular today as they used to be, and have been substituted by new models to perform various applications ( e.g., autoencoders for unsupervised learning, generative adversarial networks (GAN) for generative modes [66], variational autoencoders (VAE) [67]). However, disregarding the restricted use of these networks in this era, their influence on the advancement of neural 


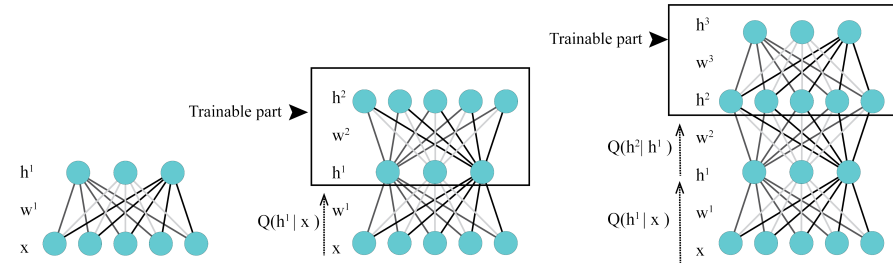

Fig. 6: Overall block diagram of a DBN used for ASD detection.

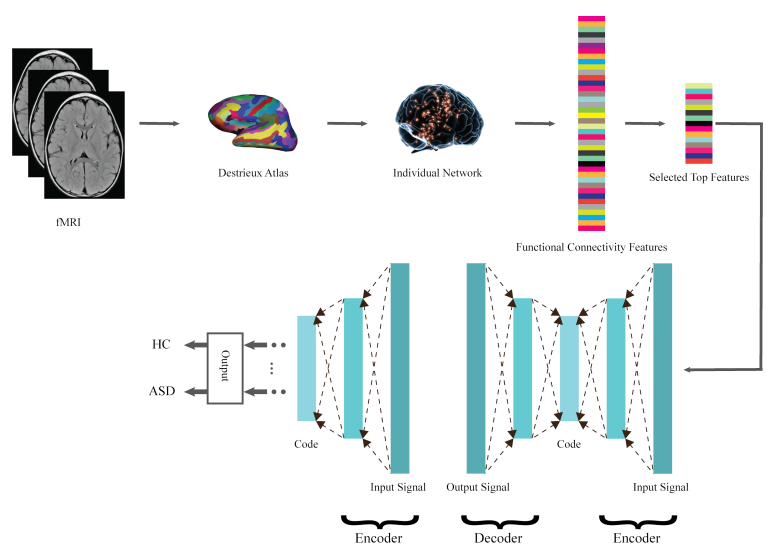

Fig. 7: Overall block diagram of an AE used for ASD detection.

networks cannot be overlooked. The use of these networks in this paper is related to the feature extraction without a supervisor or pre-training of networks. These networks serve as unsupervised, consisting of several layers after the input layer, which are shown in Figure 6 . The training of these networks is done greedily and from bottom to top, in other words, each separate layer is trained and then the next layer is appended. After training, these networks are used as a feature extractor or the network weights are used as initial weights of a network for classification [21].

3) Autoencoders (AEs): Autoencoders (AEs) are more than 30 years old, and have undergone dramatic changes over the years to enhance their performance. But the overall structure of these networks has remained the same [21].These networks consist of two parts: coder and decoder so that the first part of the input leads to coding in the latent space, and the decoder part endeavors to convert the code into preliminary data (Figure 7). Autoencoders are a special type of feedforward neural networks where the input is the same as the output. They compress the input into a lower-dimensional code and then reconstruct the output from this representation. The code is a compact "summary" or "compression" of the input, also called the latent-space representation. Various methods have been proposed to block the data memorization by the network, including sparse AE (SpAE) and denoising AE (DAE) [21]. Trained properly, the coder part of an Autoencoder can be used to extract features; creating an unsupervised feature extractor.

4) Recurrent Neural Networks (RNNs): In convolution networks, a kind of spatial dependencies in the data is addressed. But interdependencies between data are not confined to this model. For example in time-series, dependencies may be

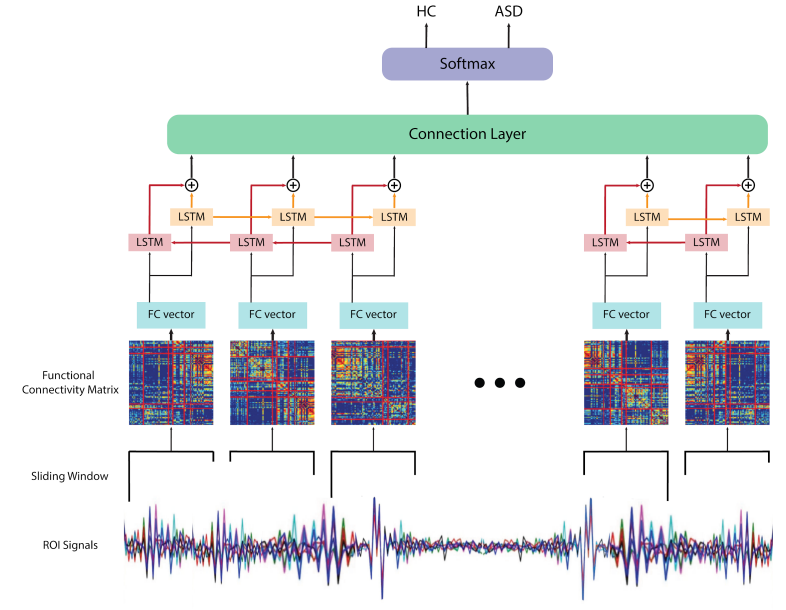

Fig. 8: Overall block diagram of an LSTM used for ASD detection.

highly distant from each other, on the other hand, the longterm and variable length of these sequences results in that the ordinary networks do not perform well enough to process these data. To overcome these problems, RNNs can be used. LSTM structures are proposed to extract long term and short term dependencies in the data (Figure 8). Another well-known structure called GRU is developed after LSTM, and since then, most efforts have been made to enhance these two structures and make them resistant to challenges (e.g., GRU-D [68] is used to find the lost data).

5) $C N N-R N N$ : The initial idea in these networks is to utilize convolution layers to amend the performance of RNNs so that the advantages of both networks can be used; CNN-RNN, on the one hand, can find temporal dependencies with the aid of RNN, and on the other hand, it can discover spatial dependencies in data with the help of convolution layers [69]. These networks are highly beneficial for analyzing time series with more than one dimension (such as video) [70] but further to the simpler matter, these networks also yield the analysis of three-dimensional data so that instead of a more complex design of a 3D-CNN, a 2D-CNN with an RNN is occasionally used. The superiority of this model is due to the feasibility of employing pre-trained models. Figure 9 demonstrates the CNN-RNN model.

6) $C N N-A E$ : In the construction of these networks, the principal aim and prerequisite have been to decrease the number of parameters. As shown before, changing merely the network layers to convolution markedly lessens the number of parameters; combining $\mathrm{AE}$ with convolution structures also makes significant contribution. This helps to exploit higher dimensional data and extracts more information from the data without changing the size of the database. Similar structures, with or without some modifications, are widely deployed for image segmentation [71], and likewise unsupervised network can be applied for network pre-training or feature extraction. Figure 10 depicts the CNN-AE network used for ASD detection. Tables I and II provide the summary of papers published on detection and rehabilitation of ASD patients using DL, 


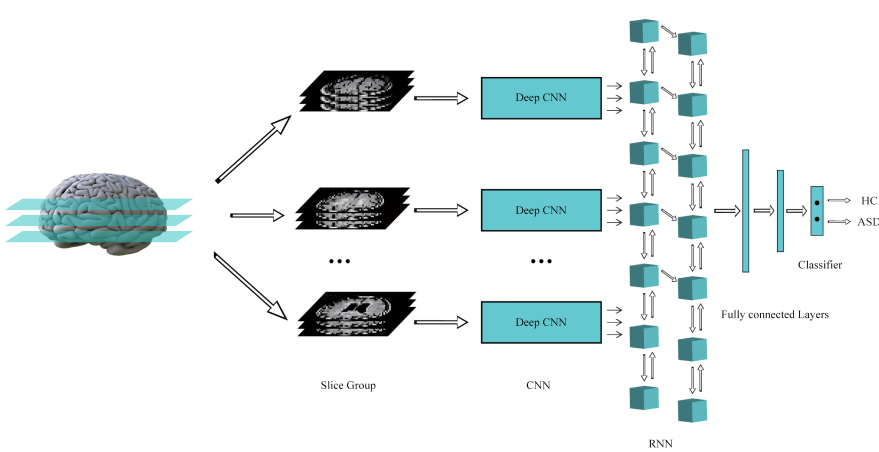

Fig. 9: Overall block diagram of a CNN-RNN used for ASD detection.

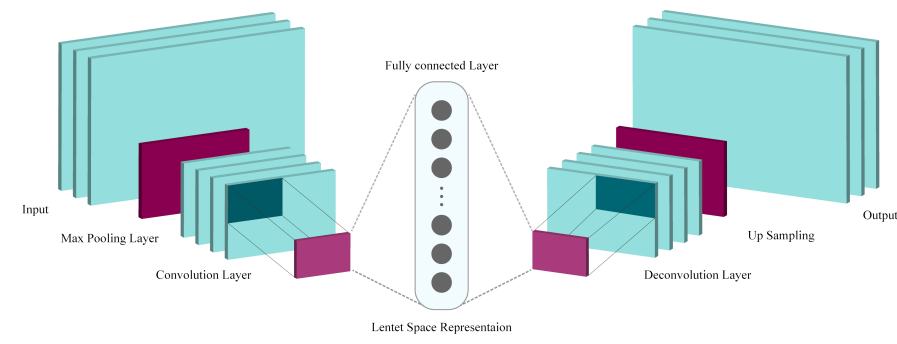

Fig. 10: Overall block diagram of a CNN-AE used for ASD detection.

respectively.

\section{DeEp LeARning TeChNiQues For ASD REHABILITATION}

Rehabilitation tools are employed in multiple fields of medicine and their main purpose is to help the patients to recover after the treatment. Various and multiple rehabilitation tools using DL algorithms have been presented. Rehabilitation tools are used to help ASD patients using mobile, computer applications, robotic devices, cloud systems, and eye tracking, which will be discussed below. Also, the summary of papers published on rehabilitation of ASD patients using DL algorithms are shown in Table III

\section{A. Mobile and Software Applications}

Facial expressions are a key mode of non-verbal communication in children with ASD and play a pivotal role in social interactions. Use of BCI systems provides insight into the user's inner-emotional state. Valles et al. [72] conducted research focused on mobile software design to provide assistance to children with ASD. They aimed to design a smart iOS app based on facial images according to Figure 11. In this way, people's faces at different angles and brightness are first photographed, and are turned into various emoji so that the autistic child can express his/her feelings and emotions. In this group's investigation [72], Kaggle's (The Facial Expression Recognition 2013) and KDEF (Kaggle's FER2013 and Karolinska Directed Emotional Faces) databases were used to train the VGG-16. In addition, the LEAP system was adapted to train the model at the University of Texas. The research provided the highest rate accuracy of $86.44 \%$. In

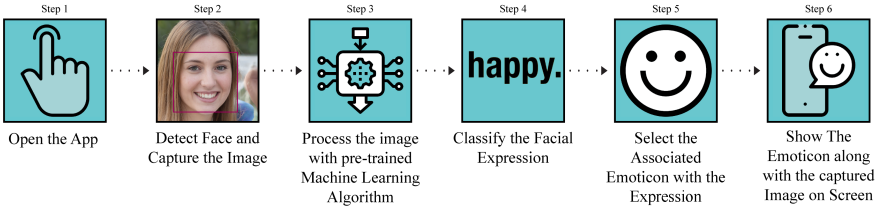

Fig. 11: Block diagram of ios application for ASD rehabilitation.

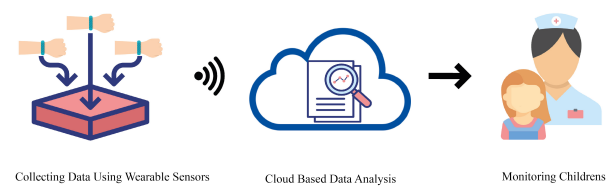

Fig. 12: Cloud system design for ASD rehabilitation.

another similar study, they achieved an accuracy of $78.32 \%$ [73].

\section{B. Cloud Systems}

Mohammadian et al. [74] proposed a new application of DL to facilitate automatic stereotypical motor movement (SMM) identification by applying multi-axis inertial measurement units (IMUs). They applied CNN to transform multi-sensor time series into feature space. An LSTM network was then combined with CNN to obtain the temporal patterns for SMM identification. Finally, they employed the classifier selection voting approach to combine an ensemble of the best base learners. After various experiments, the superiority of their proposed procedure over other base methods was proven. Figure 12 shows the real-time SMM detection system. First, IMUs, which are wearable sensors, are used for data collection; the data can then be analyzed locally or remotely (using Wi-Fi to transfer data to tablets, cell phones, medical center servers, etc.) to identify SMMs. If abnormal movements are detected, an alarm will be sent to a therapist or parents.

\section{Eye Tracking}

$\mathrm{Wu}$ et al. [75] proposed a model of DL saliency prediction for autistic children. They used DCN in their proposed paradigm, with a SM saliency map output. The fixation density map (FDM) was then processed by the single-side clipping (SSC) to optimize the proposed loss function as a true label along with the SM saliency map. Finally, they exploited an autism eye-tracking dataset to test the model. Their proposed model outperformed other base methods. Elbattah et al. [76] aimed to combine unsupervised clustring algorithms with deep learning to help ASD rehabilitation. The first step involved the visualization of the eye-tracking path, and the images captured from this step were fed to an autoencoder to learn the features. Using autoencoder features, clustering models are developed using the K-Means algorithm. Their method performed better than other state-of-art techniques. 
TABLE I: Summary of articles published using DL methods for neuroimaging-based ASD detection.

\begin{tabular}{|c|c|c|c|c|c|c|c|c|c|c|c|c|c|c|}
\hline Work & Datasets & $\begin{array}{l}\text { Neuroimaging } \\
\text { Modalities }\end{array}$ & $\begin{array}{l}\text { Number } \\
\text { of Cases }\end{array}$ & Pipelines & $\begin{array}{l}\text { Image } \\
\text { Atlas }\end{array}$ & $\begin{array}{l}\text { Preprocessing } \\
\text { Toolbox }\end{array}$ & $\begin{array}{l}\text { High level } \\
\text { Preprocessing }\end{array}$ & $\begin{array}{l}\text { Inputs } \\
\text { DNN }\end{array}$ & $\begin{array}{l}\text { DNN } \\
\text { Toolbox }\end{array}$ & DNN & $\begin{array}{c}\text { Number of } \\
\text { Layers }\end{array}$ & Classifier & K fold & $\begin{array}{l}\text { Performance } \\
\text { Criteria (\%) }\end{array}$ \\
\hline 241] & Clinical Acquisition & $\begin{array}{l}\text { T-fMRI } \\
\text { residual } \\
\text {-fMRI }\end{array}$ & $\begin{array}{l}82 \mathrm{ASD} \\
48 \mathrm{HC}\end{array}$ & $\mathrm{NA}$ & MNI152 & FSL & sw & $\begin{array}{l}\text { Single Mean Channel Input } \\
\text { Single STD Channel Input } \\
\text { Combined 2-Channel Input } \\
\end{array}$ & $\mathrm{NA}$ & $2 \mathrm{CC} 3 \mathrm{D}$ & 17 & $\begin{array}{c}\text { Majority } \\
\text { Voting } \\
\end{array}$ & $\mathrm{NA}$ & F1-Score $=89$ \\
\hline 771 & Clinical Acquisition & T-fMRI & $\begin{array}{l}82 \mathrm{ASD} \\
48 \mathrm{HC}\end{array}$ & $\mathrm{NA}$ & AAL & $\mathrm{NA}$ & $\begin{array}{l}\text { SVE, C-SVE, H-SVE, Monte } \\
\text { Carlo Approximation }\end{array}$ & $\begin{array}{l}\text { Mean-Channel Sequence } \\
\text { STD-Channel }\end{array}$ & $\mathrm{NA}$ & 2CC3D & 14 & Sigmoid & $\mathrm{NA}$ & $\mathrm{Acc}=97.32$ \\
\hline 788] & $\begin{array}{c}\text { HCP Dataset in } \\
\text { the HAFNI Project }\end{array}$ & $\begin{array}{c}\text { T-fMRI } \\
\text { rs-fMRI }\end{array}$ & $\begin{array}{l}68 \text { Subjects with } \\
7 \text { Tasks and } \\
1 \text { rs-fMRI Data }\end{array}$ & $\mathrm{NA}$ & $\mathrm{NA}$ & FSL & $\begin{array}{l}\text { Dictionary Learning } \\
\text { and Sparse Coding }\end{array}$ & Functional RNSs Maps & $\mathrm{NA}$ & 3D-CNN & 8 & Softmax & $\mathrm{NA}$ & $\mathrm{Acc}=94.61$ \\
\hline 59] & Clinical Acquisition & T-fMRI & $\begin{array}{l}21 \mathrm{ASD} \\
19 \mathrm{HC}\end{array}$ & $\mathrm{NA}$ & AAL & FSL & DA & ROIs Time-Series & Keras & LSTM & 7 & Sigmoid & 10 & Acc $=69.8$ \\
\hline 79] & Different Datasets & $\begin{array}{c}\text { T-fMRI } \\
\text { rs-fMRI } \\
\text { Phenotypic Info } \\
\end{array}$ & $\begin{array}{ll}1711 \mathrm{ASD} \\
15903 \mathrm{HC} \\
\end{array}$ & $\mathrm{NA}$ & AAL & \begin{tabular}{|c|} 
SPM \\
SpeedyPP \\
\end{tabular} & $\begin{array}{l}\text { Wavelet Transform and } \\
\text { Different Techniques } \\
\end{array}$ & FCM & Keras & 2D-CNN & 14 & Softmax & $\mathrm{NA}$ & $\begin{array}{c}\text { Ensemble AUROC }=0.92 \\
\text { Ensemble Acc }=85.19\end{array}$ \\
\hline 801 & $\begin{array}{l}\text { Clinical } \\
\text { Acquisition } \\
\end{array}$ & T-fMRI & $\begin{array}{l}82 \mathrm{ASD} \\
48 \mathrm{HC} \\
\end{array}$ & $\mathrm{NA}$ & AAL & Neurosynth & $\begin{array}{c}\text { SW } \\
\text { Corrupting Strategy }\end{array}$ & $\begin{array}{l}\text { Original fMRI Sequence } \\
\text { Mean-Channel Sequence } \\
\end{array}$ & $\mathrm{NA}$ & 2CC3D & 16 & Sigmoid & $\mathrm{NA}$ & $\mathrm{Acc}=87.1$ \\
\hline 801 & ABIDE-I & rs-fMRI & $\begin{array}{l}41 \mathrm{ASD} \\
54 \mathrm{HC}\end{array}$ & $\mathrm{NA}$ & AAL & FSL & $\begin{array}{l}\text { Prediction Distribution } \\
\text { Analysis }\end{array}$ & $\begin{array}{c}\text { std-channel sequence } \\
\text { Corrupt a ROI of Original Image }\end{array}$ & $\mathrm{NA}$ & 2CC3D & 16 & Sigmoid & $\mathrm{NA}$ & Acc $=85.3$ \\
\hline 81$]$ & $\begin{array}{l}\text { ABIDE-I } \\
\text { ABIDE II } \\
\end{array}$ & rs-fMRI & $\begin{array}{l}379 \mathrm{ASD}, 395 \mathrm{HC} \\
163 \mathrm{ASD}, 230 \mathrm{HC} \\
\end{array}$ & CPAC & $\begin{array}{c}\text { All Atlases } \\
\text { ABIDE }\end{array}$ & $\mathrm{NA}$ & $\mathrm{FCM}$ & $\begin{array}{l}\text { Concatenating Voxel-Level Maps } \\
\text { of Connectivity Fingerprints }\end{array}$ & $\mathrm{NA}$ & 3D-CNN & 7 & Sigmoid & 10 & $\mathrm{Acc}=73.3$ \\
\hline 821] & ABIDE-I & rs-fMRI & $\begin{array}{l}505 \mathrm{ASD} \\
530 \mathrm{HC} \\
\end{array}$ & CPAC & \begin{tabular}{|c|} 
CC-200 \\
AAL \\
Dosenbach160 \\
\end{tabular} & $\mathrm{NA}$ & FCM, DA & $\begin{array}{c}\text { Masking } \\
\text { Correlations } \\
\end{array}$ & PyTorch & $\mathrm{AE}$ & $\mathrm{NA}$ & SLP & 10 & $\begin{array}{c}\text { Acc }=70.1 \\
\text { Sen=67.8 } \\
\text { Spec }=72.8 \\
\end{array}$ \\
\hline 831 & ABIDE-I & rss-fMRI & 872 subjects & CPAC & HO & $\begin{array}{l}\text { Nilearn } \\
\end{array}$ & $\mathrm{NA}$ & Raw Images & $\mathrm{NA}$ & G-CNNs & 5 & Softmax & 10 & Acc=70.86 \\
\hline 841 & ABIDE-I & rs-fMRI & $\begin{array}{l}474 \mathrm{ASD} \\
539 \mathrm{HC} \\
\end{array}$ & cCS & AAL & $\mathrm{NA}$ & FCM & $\begin{array}{c}\text { Functional } \\
\text { Connectomes }\end{array}$ & $\mathrm{NA}$ & $\begin{array}{l}\text { BrainNetClNN } \\
\text { with Proposed } \\
\text { Layers }\end{array}$ & 15 & Softmax & 5 & $\begin{array}{l}\text { Acc= } 68.7 \\
\text { Sen }=69.2 \\
\text { Spe }=68.3\end{array}$ \\
\hline 85] & ABIDE-I & rss-fMRI & $\begin{array}{l}13 \mathrm{ASD} \\
22 \mathrm{HC}\end{array}$ & $\mathrm{NA}$ & AAL & \begin{tabular}{|c|} 
SPM8 \\
REST \\
DPARSF \\
\end{tabular} & $\begin{array}{c}\text { Qcut, NMI } \\
\text { Statistic Matrix }\end{array}$ & 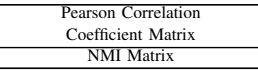 & $\mathrm{NA}$ & DAE & $\mathrm{NA}$ & $\mathrm{NA}$ & $\mathrm{NA}$ & $\mathrm{Acc}=54.49$ \\
\hline 86 & ABIDE & rss-fMRI & $\begin{array}{l}11 \mathrm{ASD} \\
16 \mathrm{HC}\end{array}$ & $\mathrm{NA}$ & $\mathrm{NA}$ & $\begin{array}{l}\text { FSL } \\
\text { BET }\end{array}$ & $\begin{array}{l}\text { Convert NII Files } \\
\text { to PNG Images }\end{array}$ & $\begin{array}{l}\text { Preprocessed } \\
\text { PNG Images }\end{array}$ & Caffe & LeNet-5 & Standard & Softmax & $\mathrm{NA}$ & $\begin{array}{l}\text { Acc=100 } \\
\text { Sen=99.99 } \\
\text { Spec }=100\end{array}$ \\
\hline 87] & ABIDE-I & rs-fMRI & $\begin{array}{l}55 \mathrm{ASD} \\
55 \mathrm{HC}\end{array}$ & NIAK & AAL & $\mathrm{NA}$ & $\begin{array}{l}\text { FCM, Feature } \\
\text { Selection }\end{array}$ & Whole-Brain FCPs & $\mathrm{NA}$ & $\begin{array}{l}\text { Multiple } \\
\text { SAEs }\end{array}$ & 4 & $\begin{array}{c}\text { Softmax } \\
\text { regression }\end{array}$ & 5 & Acc $=86.36$ \\
\hline 621 & $\begin{array}{c}\text { ABIDE-I } \\
\text { ABIDE-II } \\
\text { ABIDE-I + II }\end{array}$ & rs-fMRI & $\begin{array}{l}54 \text { ASD } \\
62 \mathrm{HC} \\
156 \mathrm{ASD} \\
187 \mathrm{HC}\end{array}$ & $\mathrm{NA}$ & $\mathrm{NA}$ & SPM8 & $\begin{array}{c}\text { Dimension Reduction } \\
\text { FFT }\end{array}$ & $\begin{array}{l}\text { Images with } 95 \times 68,79 \times 68 \text {, } \\
\text { and } 79 \times 95 \text { Dimensions, Around } \\
\text { the } x, y \text {, and } z \text { Axes }\end{array}$ & $\begin{array}{c}\text { Keras } \\
\text { with } \\
\text { Theano } \\
\text { backend }\end{array}$ & MCNNES & 9 & Binary SR & 10 & $\begin{array}{c}\text { Acc }=72.73 \\
\text { Sen=71.2 } \\
\text { Spec }=73.48\end{array}$ \\
\hline 88 & ABIDE & rss-fMRI & $\begin{array}{l}542 \mathrm{ASD} \\
625 \mathrm{HC}\end{array}$ & CPAC & $\begin{array}{c}\text { All } \\
\text { Atlases }\end{array}$ & $\mathrm{NA}$ & $\begin{array}{l}\text { Creating Stochastic } \\
\text { Parcellations by Poisson } \\
\text { Disk Sampling }\end{array}$ & $\begin{array}{c}\text { Gray Matter } \\
\text { Mask Parcellations }\end{array}$ & $\mathrm{NA}$ & 3D-CNN & 6 & $\begin{array}{l}\text { Various } \\
\text { Methods }\end{array}$ & 10 & Acc $=72$ \\
\hline 89$]$ & ABIDE-I & rs-fMRI & $\begin{array}{l}465 \mathrm{ASD} \\
507 \mathrm{HC}\end{array}$ & DPARSF & AAL & $\mathrm{NA}$ & FCM & $\begin{array}{c}\text { Edge Weights of Subjects' } \\
\text { Brain Graph }\end{array}$ & Keras & VAE & 3 & $\mathrm{NA}$ & $\mathrm{NA}$ & NA \\
\hline 901] & ABIDE-I & rs-fMRI & $\begin{array}{l}539 \mathrm{ASD} \\
573 \mathrm{HC}\end{array}$ & CCS & $\begin{array}{l}\text { Craddock } \\
200\end{array}$ & Neurosynth & $\mathrm{DA}$ & $\begin{array}{l}\text { Mean Time Courses } \\
\text { from ROIs }\end{array}$ & Keras & LSTM & 5 & Sigmoid & 10 & Acc $=68.5$ \\
\hline 91] & ABIDE & $\begin{array}{l}\text { rs-fMRI, } \\
\text { Phenotypic } \\
\text { Info }\end{array}$ & $\begin{array}{l}505 \mathrm{ASD} \\
530 \mathrm{HC}\end{array}$ & $\mathrm{NA}$ & СC200 & DPABI & $\begin{array}{c}\text { Slicetiming, Spatial } \\
\text { Standardization, Smoothing, } \\
\text { Filtering, Removing } \\
\text { Covariates, FCM, AE-MKFC }\end{array}$ & $\begin{array}{l}\text { 4005-Dimensional } \\
\text { Eigenvector }\end{array}$ & $\mathrm{NA}$ & $\mathrm{SAE}$ & 3 & Clustering & $\mathrm{NA}$ & $\begin{array}{c}\text { Acc }=61 \\
\text { F1-Score }=60.2\end{array}$ \\
\hline 92] & ABIDE & rs-fMRI & $\begin{array}{l}42 \mathrm{ASD} \\
42 \mathrm{HC}\end{array}$ & $\mathrm{NA}$ & $\mathrm{NA}$ & FSL & $\begin{array}{l}\text { Independent Components } \\
\text { (Time Course, Power } \\
\text { Spectrum and Spatial Map) }\end{array}$ & $\begin{array}{l}\text { Time Courses of } \\
\text { Each Subject }\end{array}$ & $\mathrm{NA}$ & SAE & 9 & Softmax & 21 & $\begin{array}{l}\text { Acc }=87.21 \\
\text { Sen=89.49 } \\
\text { Spec=83.73 }\end{array}$ \\
\hline 93] & ABIDE-I & rs-fMRI & $\begin{array}{l}\text { NY site } \\
\text { UM site } \\
\text { US site } \\
\text { UC site }\end{array}$ & CCS & AAL & Neurosynth & $\mathrm{DA}$ & $\begin{array}{l}\text { fMRI ROI Time-Series, } \\
\text { Functional Connectivity }\end{array}$ & Keras & LSTM & 6 & Sigmoid & 10 & Acc $=74.8$ \\
\hline 94] & ABIDE-I & rs-fMRI & $\begin{array}{l}408 \mathrm{ASD} \\
401 \mathrm{HC}\end{array}$ & CPAC & $\begin{array}{l}\text { HO } \\
\text { AAL } \\
\text { CC200 }\end{array}$ & FSL & $\mathrm{NA}$ & $\begin{array}{l}3 \text { Different FCM+ } \\
\text { Demographic Data }\end{array}$ & Keras & DANN & 25 & Sigmoid & 10 & $\begin{array}{l}\text { Acc=73.2. } \\
\text { Sen=74.5 } \\
\text { Spec }=71.7\end{array}$ \\
\hline 951 & ABIDE & rs-fMRI & $\begin{array}{l}\text { At Least } 60 \\
\text { Subjects }\end{array}$ & CCS & AAL & FSL & $\begin{array}{l}\text { DTL-NN Framework: } \\
\text { Offline Learning, Transfer } \\
\text { Learning FCM }\end{array}$ & FC Patterns & $\mathrm{NA}$ & SSAE & 4 & $\begin{array}{c}\text { Softmax } \\
\text { regression }\end{array}$ & 5 & $\begin{array}{c}\text { Avg Acc }=67.1 \\
\text { Avg Sen=65.7 } \\
\text { Avg spec }=68.3 \\
\text { AUC }=0.71\end{array}$ \\
\hline 96] & ABIDE I+II & rs-fMRI & $\begin{array}{l}993 \mathrm{ASD} \\
1092 \mathrm{HC}\end{array}$ & $\mathrm{NA}$ & $\begin{array}{c}\text { AAL } \\
\text { Schaefer-100 } \\
\text { HO } \\
\text { Schaefer-400 }\end{array}$ & $\begin{array}{c}\text { FAST } \\
\text { BET } \\
\text { FAST }\end{array}$ & $\mathrm{NA}$ & $\begin{array}{l}\text { Mean Time-Series } \\
\text { within Each ROI }\end{array}$ & $\mathrm{NA}$ & 1D-CNN & 5 & Softmax & 10 & Acc $=68$ \\
\hline
\end{tabular}




\begin{tabular}{|c|c|c|c|c|c|c|c|c|c|c|c|c|c|c|}
\hline 97] & ABIDE-I & rs-fMRI & $\begin{array}{l}529 \mathrm{ASD} \\
573 \mathrm{HC}\end{array}$ & $\begin{array}{c}\text { All } \\
\text { Pipelines }\end{array}$ & $\mathrm{NA}$ & $\mathrm{NA}$ & $\begin{array}{l}\text { Single Volume } \\
\text { Image Generator }\end{array}$ & $\begin{array}{l}\text { Glass Brain and } \\
\text { Stat Map Images }\end{array}$ & Keras & $\begin{array}{l}4 \text { Deep } \\
\text { Ensemble } \\
\text { Classifier } \\
\text { techniques }\end{array}$ & 16 & Sigmoid & $\mathrm{NA}$ & $\begin{array}{c}\text { Acc }=87 \\
\text { F1-score }=86 \\
\text { Recall }=85.2 \\
\text { Pre }=86.8\end{array}$ \\
\hline [981] & ABIDE-II & rs-fMRI & $303 \mathrm{ASD}, 390 \mathrm{HC}$ & $\mathrm{NA}$ & $\mathrm{NA}$ & FSL & $\mathrm{NA}$ & ID Time Series from Voxels & $\mathrm{NA}$ & ID-CAE & 14 & $\mathrm{NA}$ & $\mathrm{NA}$ & Acc $=65.3$ \\
\hline 99] & ABIDE & rs-fMRI & $40 \mathrm{ASD}, 40 \mathrm{HC}$ & CCS & $\mathrm{NA}$ & $\mathrm{NA}$ & $\begin{array}{c}\text { Thresholding Based } \\
\text { Segmentation }\end{array}$ & WM, GM, CSF & $\mathrm{NA}$ & AlexNet & Standard & Softmax & $\mathrm{NA}$ & Acc $=82.61$ \\
\hline 1000 & ABIDE & rs-fMRI & $\begin{array}{l}\text { Whole } \\
\text { Dataset }\end{array}$ & $\begin{array}{c}\text { All } \\
\text { Pipelines }\end{array}$ & $\begin{array}{l}\text { Parcellated } \\
\text { into 200 } \\
\text { Regions }\end{array}$ & $\mathrm{NA}$ & $\begin{array}{l}\text { DA Using SMOTE } \\
\text { and Graph Network } \\
\text { Motiff, FCM }\end{array}$ & $\begin{array}{l}\text { Upper Triangle Part of } \\
\text { the Correlation Matrix }\end{array}$ & $\mathrm{NA}$ & $\begin{array}{c}\text { ASD- } \\
\text { DiagNet }\end{array}$ & Proposed & SLP & $\mathrm{NA}$ & $\begin{array}{l}\mathrm{Acc}=82 \\
\mathrm{Sen=79.1} \\
\mathrm{Spec}=83.3\end{array}$ \\
\hline 601 & ABIDE-I & rs-fMRI & $\begin{array}{l}12 \mathrm{ASD} \\
14 \mathrm{HC}\end{array}$ & CPAC & $\operatorname{scsc}$ & $\mathrm{NA}$ & $\begin{array}{l}\text { Time Series Extraction } \\
\text { from Different Regions, } \\
\text { Connectivity Martix, } \\
\text { SMOTE Algorithm }\end{array}$ & FCM & PyTorch & $\begin{array}{c}\text { Auto- } \\
\text { ASD- } \\
\text { Network }\end{array}$ & Proposed & SVM & 5 & $\begin{array}{l}\text { Acc=80 } \\
\text { Sen=73 } \\
\text { Spec }=83\end{array}$ \\
\hline 61] & ABIDE-I & rss-fMRI & $\begin{array}{l}505 \mathrm{ASD} \\
530 \mathrm{HC}\end{array}$ & CPAC & CC400 & $\mathrm{NA}$ & FCM & FCM & $\mathrm{NA}$ & 2D-CNN & 20 & MLP & 10 & $\begin{array}{l}\text { Acc }=70.20 \\
\text { Sen }=77.00 \\
\text { Spec }=61.00\end{array}$ \\
\hline 101] & ABIDE & rs-fMRI & $\begin{array}{l}505 \mathrm{ASD} \\
530 \mathrm{HC}\end{array}$ & $\mathrm{NA}$ & $\mathrm{NA}$ & $\mathrm{NA}$ & FCM & 1D-Vector of FCM & $\mathrm{NA}$ & $\begin{array}{c}1 \mathrm{D} \mathrm{CNN} \\
-\mathrm{AE}\end{array}$ & 7 & Softmax & $\mathrm{NA}$ & $\begin{array}{l}\text { Acc=70 } \\
\text { Sen=74 } \\
\text { Spec }=63\end{array}$ \\
\hline 102] & ABIDE-I & rs-fMRI & $\begin{array}{l}539 \mathrm{ASD} \\
573 \mathrm{HC}\end{array}$ & $\mathrm{NA}$ & $\mathrm{NA}$ & FreeSurfer & $\mathrm{NA}$ & Single 3D Image & Theano & 3D-FCNN & 13 & Softmax & 6 & $\begin{array}{l}\text { Mean DSC }=91.56 \\
\text { Mean MHD }=14.05\end{array}$ \\
\hline 103] & ABIDE-I & rs-fMRI & $\begin{array}{l}501 \mathrm{ASD} \\
553 \mathrm{HC}\end{array}$ & DPARSF & AAL & $\mathrm{NA}$ & $\begin{array}{l}\text { FCM, Converting to } \\
\text { ID-Vector }\end{array}$ & $\begin{array}{l}1000 \text { Features Selected } \\
\text { by the SVM-RFE }\end{array}$ & $\mathrm{NA}$ & SSAE & 3 & Softmax & $\begin{array}{c}\text { Different } \\
\text { Folds }\end{array}$ & $\begin{array}{l}\text { Acc }=93.59 \\
\text { Sen=92.52 } \\
\text { Spec }=94.56\end{array}$ \\
\hline [104] & ABIDE-I & rss-fMRI & $\begin{array}{l}100 \mathrm{ASD} \\
100 \mathrm{HC}\end{array}$ & $\mathrm{NA}$ & $\mathrm{NA}$ & $\begin{array}{l}\text { FSL } \\
\text { FEAT }\end{array}$ & $\begin{array}{c}\text { Online Dictionary Learning } \\
\text { and Sparse Representation } \\
\text { Techniques, Generating } \\
\text { Spatial Overlap Patterns }\end{array}$ & $\begin{array}{l}\text { 4D Matrix with 150 3D } \\
\text { Network Overlap Maps }\end{array}$ & Theano & 3D-CNN & 14 & $\mathrm{NA}$ & 10 & $\begin{array}{l}\text { Average Acc }=70.5 \\
\text { Average } \operatorname{Sen}=74 \\
\text { Average } \mathrm{Spec}=67\end{array}$ \\
\hline 105] & ABIDE-I & $\begin{array}{c}\text { rs-fMRI \& } \\
\text { Phenotypic Info }\end{array}$ & $\begin{array}{l}529 \mathrm{ASD} \\
571 \mathrm{HC}\end{array}$ & CPAC & но & FSL & $\begin{array}{l}\text { Population Graph Construction, } \\
\text { Feature Selection Strategies } \\
\text { (RFE, PCA, MLP, AE) }\end{array}$ & $\begin{array}{l}\text { Population } \\
\text { Graph }\end{array}$ & $\begin{array}{l}\text { Scikit } \\
\text {-learn }\end{array}$ & GCN & 11 & Softmax & 10 & Acc $=80.0$ \\
\hline 106 & ABIDE-I & $\begin{array}{c}\text { rs-fMRI \& } \\
\text { Phenotypic Info }\end{array}$ & $\begin{array}{l}403 \mathrm{ASD} \\
468 \mathrm{HC}\end{array}$ & cCS & CC200 & $\mathrm{NA}$ & $\mathrm{DA}$ & $\begin{array}{l}\text { Mean Time-Series } \\
\text { from ROIs }\end{array}$ & Keras & LSTM & 6 & Sigmoid & 10 & Acc $=70.1$ \\
\hline 107] & ABIDE-I & $\begin{array}{c}\text { rs-fMRI \& } \\
\text { S-MRI \& } \\
\text { Phenotypic } \\
\text { Info }\end{array}$ & $\begin{array}{l}505 \mathrm{ASD} \\
530 \mathrm{HC}\end{array}$ & CPAC & $\begin{array}{l}\text { Craddock } \\
200\end{array}$ & $\mathrm{NA}$ & FCM & 1D-Vector of FCM & $\mathrm{NA}$ & $\begin{array}{l}\text { Two } \\
\text { SdAE } \\
+ \text { MLP }\end{array}$ & $\mathrm{NA}$ & Softmax & 10 & $\begin{array}{l}\text { Acc=70 } \\
\text { Sen=74 } \\
\text { Spec }=63\end{array}$ \\
\hline 108] & Clinical Acquisition & \begin{tabular}{|l} 
rs-fMRI \\
Fetal \\
BOLD fMRI \\
\end{tabular} & $\begin{array}{l}75 \text { Qualified } \\
\text { Subjects }\end{array}$ & $\mathrm{NA}$ & $\mathrm{NA}$ & $\begin{array}{c}\text { FSL } \\
\text { Brainsuite } \\
\text { SPM8 } \\
\text { CONN } \\
\end{array}$ & $\begin{array}{l}\text { Extraction of Fetal } \\
\text { Brain fMRI Data, SW }\end{array}$ & $\begin{array}{l}\text { Mean Time Series } \\
\text { of 3D fMRI Volumes }\end{array}$ & PyTorch & $3 \mathrm{D}-\mathrm{CNN}$ & 7 & Sigmoid & $\mathrm{NA}$ & $\begin{array}{l}\text { F1-score }=84 \\
\text { AUC }=91\end{array}$ \\
\hline 109] & $\begin{array}{l}\text { ABIDE-I } \\
\text { ABIDE-II }\end{array}$ & $\begin{aligned} \text { rs-fMRI } \\
\text { s-MRI }\end{aligned}$ & $\begin{array}{l}116 \mathrm{ASD} \\
69 \mathrm{HC}\end{array}$ & $\mathrm{NA}$ & AAL & SPM8 & $\begin{array}{l}\text { Segmentation, Average } \\
\text { Mean Time Series } \\
\text { of Each ROI }\end{array}$ & $\begin{array}{l}\text { Rs-fMRI + GM+WM } \\
\text { Data Fusions }\end{array}$ & Theano & DBN & 6 & LR & 10 & $\begin{array}{l}\text { Acc }=65.56 \\
\text { Sen=84 } \\
\text { Spec }=32.96\end{array}$ \\
\hline [110] & IMPAC & $\begin{array}{c}\text { rs-fMRI } \\
\text { s-MRI }\end{array}$ & $\begin{array}{l}418 \mathrm{ASD} \\
497 \mathrm{HC}\end{array}$ & $\mathrm{NA}$ & $\begin{array}{l}\text { All } \\
\text { atlases }\end{array}$ & $\mathrm{NA}$ & $\begin{array}{c}\text { FCM, Features } \\
\text { Extraction from S-MRI }\end{array}$ & $\begin{array}{c}\text { FCM Vector } \\
\text { Anatomical Features } \\
\text { Combination of Both } \\
\text { Anatomical and Connectivity } \\
\text { ACM Vector }\end{array}$ & $\begin{array}{l}\text { Keras } \\
\text { Tensor- } \\
\text { Flow } \\
\text { Caffe }\end{array}$ & $\begin{array}{l}\text { Different } \\
\text { Networks }\end{array}$ & 8 & $\begin{array}{l}\text { Various } \\
\text { Methods }\end{array}$ & 3 & $\mathrm{AUC}=80$ \\
\hline [111] & ABIDE-I & rs-fMRI & $\begin{array}{l}368 \mathrm{ASD} \\
449 \mathrm{HC}\end{array}$ & CPAC & $\begin{array}{c}\text { AAL } \\
\text { CC200 } \\
\text { Destrieux }\end{array}$ & Freesurfer & $\begin{array}{l}\text { FCM, } \\
\text { Fisher Score }\end{array}$ & 1D-Vector of FCM & $\mathrm{NA}$ & $\begin{array}{l}\text { Ensemble } \\
\text { of } 5 \text { Stacked } \\
\text { AEs and } \\
\text { MLP }\end{array}$ & 31 & $\begin{array}{l}\text { Label Fus- } \\
\text { ion Using } \\
\text { the Average } \\
\text { of Softmax } \\
\text { Probabilities }\end{array}$ & 10 & $\begin{array}{l}\text { Acc }=85.06 \\
\text { Sen }=81 \\
\text { Spec }=89\end{array}$ \\
\hline [112] & NDAR & $\begin{array}{c}\text { rss-fMRI } \\
s-M R I \\
\end{array}$ & $\begin{array}{l}61 \mathrm{ASD} \\
215 \mathrm{HC}\end{array}$ & $\mathrm{NA}$ & $\mathrm{NA}$ & $\mathrm{NA}$ & $\begin{array}{l}\text { Data-Driven Landmark Discovery } \\
\text { Algorithm, Patch Extraction }\end{array}$ & $\begin{array}{l}50 \text { Patches Extracted } \\
\text { from } 50 \text { Landmarks }\end{array}$ & $\mathrm{NA}$ & $\begin{array}{c}\text { Multi- } \\
\text { Channel CNN }\end{array}$ & 13 & Softmax & 10 & Acc $=76.24$ \\
\hline 631 & NDAR & $\begin{array}{c}\text { All } \\
\text { Modalities }\end{array}$ & $\begin{array}{l}78 \mathrm{ASD} \\
124 \mathrm{HC}\end{array}$ & $\mathrm{NA}$ & $\begin{array}{c}\text { Proposed } \\
\text { Atlas }\end{array}$ & $\begin{array}{l}\text { FSL } \\
\text { BET }\end{array}$ & $\begin{array}{l}\text { PICA, Extraction } \\
\text { of PSD }\end{array}$ & $\begin{array}{l}\text { PSDs of } 34 \\
\text { Components }\end{array}$ & $\mathrm{NA}$ & $\begin{array}{c}34 \\
\text { SAEs }\end{array}$ & $\begin{array}{l}\text { Each } \\
\text { SAE } \\
\text { Hay 2 } \\
\text { Layers }\end{array}$ & PSVM & $\mathrm{NA}$ & $\begin{array}{l}\text { Acc=88.5 } \\
\text { Sen=85.1 } \\
\text { Spec }=90.4\end{array}$ \\
\hline 113] & NDAR & s-MRI & $60 \mathrm{ASD}, 211 \mathrm{HC}$ & $\mathrm{NA}$ & $\mathrm{NA}$ & $\begin{array}{c}\text { In-House } \\
\text { Tools }\end{array}$ & 3D Patches Extraction & Patch Size 16×64×16 & $\mathrm{NA}$ & DDUNET & $\begin{array}{c}11 \\
\text { Blocks }\end{array}$ & $\mathrm{NA}$ & 5 & $\mathrm{NA}$ \\
\hline [114] & $\begin{array}{l}\text { ABIIE-I } \\
\text { NDAR/Pitt } \\
\text { NDAR/IBIS }\end{array}$ & s-MRI & $\begin{array}{l}21 \mathrm{ASD}, 21 \mathrm{HC} \\
16 \mathrm{ASD}, 16 \mathrm{HC} \\
10 \mathrm{ASD}, 10 \mathrm{HC} \\
\end{array}$ & $\mathrm{NA}$ & $\mathrm{NA}$ & $\begin{array}{c}\text { FSL } \\
\text { iBEAT }\end{array}$ & $\begin{array}{l}\text { Segmentation, Shape } \\
\text { Feature Extraction }\end{array}$ & $\begin{array}{l}\text { CDF Values } \\
\text { of Features }\end{array}$ & $\mathrm{NA}$ & SNCAE & $\mathrm{NA}$ & Softmax & $\mathrm{NA}$ & $\mathrm{Acc}=96.88$ \\
\hline
\end{tabular}




\begin{tabular}{|c|c|c|c|c|c|c|c|c|c|c|c|c|c|c|}
\hline 115 & ABIDE-I & s-MRI & $\begin{array}{l}78 \mathrm{ASD} \\
104 \mathrm{HC}\end{array}$ & $\mathrm{NA}$ & Destrieux & FreeSurfer & $\begin{array}{l}\text { Construction of Individual } \\
\text { Network, F-score }\end{array}$ & 3000 Top Features & $\mathrm{NA}$ & SAE & 3 & Softmax & 10 & $\begin{array}{l}\text { Acc=90.39 } \\
\text { Sen=84.37 } \\
\text { Spec=95.88 }\end{array}$ \\
\hline 116 & $\begin{array}{c}\text { HCP } \\
\text { ABIDE-I }\end{array}$ & s-MRI & $\begin{array}{l}1113 \mathrm{HC} \\
83 \mathrm{ASD} \\
105 \mathrm{HC}\end{array}$ & $\mathrm{NA}$ & $\begin{array}{c}\text { Desikan- } \\
\text { Killia }\end{array}$ & FreeSurfer & $\begin{array}{l}\text { Normalization, Apply } \\
\text { One-Hot Coding }\end{array}$ & Preprocessed Images & $\begin{array}{l}\text { Tensor- } \\
\text { Flow } \\
\text { Keras }\end{array}$ & DEA & 3 & $\mathrm{NA}$ & 10 & $\mathrm{AUC}=63.9$ \\
\hline 117 & $\begin{array}{c}\text { ABIDE } \\
\text { CombiRx }\end{array}$ & s-MRI & 1112 Subjects & NA & NA & SPM12 & $\mathrm{NA}$ & $\begin{array}{c}2 \text { Slices } \\
\text { Along Each Axial, } \\
\text { Coronal, and Sagittal }\end{array}$ & Keras & DCNN & 17 & Sigmoid & $\mathrm{NA}$ & $\begin{array}{l}\text { Acc=84 } \\
\text { Sen=77 } \\
\text { Spec }=85\end{array}$ \\
\hline [118] & ABIDE-II & s-MRI & $\mathrm{NA}$ & $\mathrm{NA}$ & DKT & FreeSurfer & Segmentation & $\begin{array}{l}\text { Coronal, Axial and Sagittal } \\
\text { 2D Slices }\end{array}$ & PyTorch & 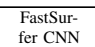 & Proposed & Softmax & $\mathrm{NA}$ & $\mathrm{NA}$ \\
\hline 119] & ABIDE-I & s-MRI & $\begin{array}{l}500 \mathrm{ASD} \\
500 \mathrm{HC}\end{array}$ & NA & $\begin{array}{c}\text { HO Cortical } \\
\text { and } \\
\text { Subcortical } \\
\text { Structural } \\
\text { Atlas } \\
\end{array}$ & FSL & $\begin{array}{l}\text { GABM Method, New } \\
\text { Chromosome Encoding } \\
\text { Scheme }\end{array}$ & $\begin{array}{l}\text { Preprocessed } \\
\text { MRI Scans }\end{array}$ & $\mathrm{NA}$ & 3D-CNN & 11 & Softmax & 5 & $\mathrm{Acc}=70$ \\
\hline 1201 & $\begin{array}{c}\text { Clinical } \\
\text { Acquisition }\end{array}$ & s-MRI & $48 \mathrm{HC}$ & NA & NA & FreeSurfer & $\begin{array}{l}\text { Sparse Annotations, } \\
\text { DA }\end{array}$ & Image Patch & Caffe & 3D-CNN & 18 & Softmax & $\mathrm{NA}$ & $\begin{array}{l}\text { Acc }=91.6 \\
\text { AUC }=94.1\end{array}$ \\
\hline 1211 & ABIDE-I & rs-fMRI & $\begin{array}{l}270 \mathrm{ASD} \\
305 \mathrm{HC}\end{array}$ & CPAC & $\begin{array}{c}\text { Brain- } \\
\text { Netome } \\
\text { Atlas } \\
\text { (BNA) }\end{array}$ & $\mathrm{NA}$ & $\begin{array}{c}\text { Filtering, Calculating } \\
\text { Mean Time Series for } \\
\text { ROIs Using BrainNetome } \\
\text { Atlas (BNA), Normalization }\end{array}$ & $\begin{array}{l}\text { Mean Time Series Data } \\
\text { Stacked Across ROIs }\end{array}$ & $\mathrm{NA}$ & $\begin{array}{l}\text { CNN- } \\
\text { GRU }\end{array}$ & 14 & Sigmoid & 5 & $\begin{array}{l}\text { Acc }=74.54 \\
\text { Sen=63.46 } \\
\text { Spec }=84.33\end{array}$ \\
\hline 1221 & $\begin{array}{c}\text { Clinical } \\
\text { Acquisition }\end{array}$ & fNIRS & $\begin{array}{l}25 \mathrm{ASD} \\
22 \mathrm{HC}\end{array}$ & No & No & No & $\begin{array}{l}\text { Transformation of } \\
\text { the Time Series } \\
\text { to Three Variants }\end{array}$ & PM, GM, SM & Keras & $\begin{array}{l}\text { 1D CNN- } \\
\text { LSTM }\end{array}$ & NA & Bagging & $\mathrm{NA}$ & $\begin{array}{l}\text { Acc=95.7 } \\
\text { Sen=97.1 } \\
\text { Spec }=94.3\end{array}$ \\
\hline [123] & $\begin{array}{c}\text { Clinical } \\
\text { Acquisition }\end{array}$ & fNIRS & $\begin{array}{l}25 \mathrm{ASD} \\
22 \mathrm{HC}\end{array}$ & No & No & No & $\begin{array}{c}\text { SW } \\
\text { Converted into the } \\
\text { 3D Tensor }\end{array}$ & 3D Tensor & $\mathrm{NA}$ & CGRNN & 7 & $\mathrm{NA}$ & $\mathrm{NA}$ & $\begin{array}{l}\text { Acc }=92.2 \\
\text { Sen=85.0 } \\
\text { Spec }=99.4\end{array}$ \\
\hline 124] & $\begin{array}{l}\text { Different } \\
\text { Datasests }\end{array}$ & s-MRI & $\mathrm{NA}$ & $\mathrm{NA}$ & $\begin{array}{l}\text { Various } \\
\text { Methods }\end{array}$ & $\begin{array}{c}\text { FreeSurfer } \\
\text { FSL } \\
\text { SPM12 } \\
\text { VolBrain }\end{array}$ & Geometric DA & 3D Cortical Mask & Theano & ConvNet & U-Nets & $\mathrm{NA}$ & 8 & $\mathrm{NA}$ \\
\hline [125] & ABIDE I+II & rs-fMRI & $\begin{array}{l}620 \mathrm{ASD} \\
2085 \mathrm{HC}\end{array}$ & CPAC & но & FSL & $\begin{array}{l}\text { Performed an Automatic Quality } \\
\text { Control, Visually Inspection, } \\
9 \text { Temporal Summary Measures, } \\
\text { Mean and STD of the Summary } \\
\text { Measures, Normalization, } \\
\text { Occlusion of Brain Regions }\end{array}$ & Each Summary Measure & $\mathrm{NA}$ & $\begin{array}{c}\text { MM- } \\
\text { ensemble } \\
\text { (3D-CNN) }\end{array}$ & 7 & $\begin{array}{l}\text { Majority } \\
\text { Voting }\end{array}$ & 5 & $\begin{array}{c}\text { Acce64 } \\
\text { F1-Score=66 }\end{array}$ \\
\hline [126] & ABIDE-I & $\begin{array}{l}\text { rs-fMRI } \\
\text { Phenotypic } \\
\text { Information }\end{array}$ & $\begin{array}{l}184 \mathrm{ASD} \\
110 \mathrm{HC}\end{array}$ & CPAC & $\mathrm{NA}$ & NA & Down Sampling & Raw 4D Volume & $\mathrm{NA}$ & $\begin{array}{l}\text { 3D-CNN } \\
\text { C-LSTM }\end{array}$ & 21 & Softmax & 5 & $\begin{array}{c}\text { Acc=77 } \\
\text { F1-score }=78\end{array}$ \\
\hline [127] & ABIDE-I & $\begin{array}{l}\mathrm{rs}-\mathrm{fMRI} \text { and } \\
\mathrm{s}-\mathrm{MRI}\end{array}$ & $\begin{array}{l}403 \mathrm{ASD} \\
468 \mathrm{HC}\end{array}$ & $\mathrm{NA}$ & 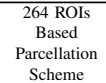 & $\begin{array}{c}\text { AFNI } \\
\text { FSL } \\
\text { MATLAB }\end{array}$ & $\begin{array}{c}\text { FCM, } \\
\text { Feature Extraction } \\
\text { (Different Features) }\end{array}$ & Normalized Features & $\mathrm{NA}$ & $\mathrm{AE}$ & 7 & DNN & 10 & $\begin{array}{l}\text { Acc }=79.2 \\
\text { AUC }=82.4\end{array}$ \\
\hline 128 & ABIDE-I & rs-fMRI & $\begin{array}{l}505 \mathrm{ASD} \\
530 \mathrm{HC}\end{array}$ & CPAC & $\mathrm{CC} 200$ & NA & $\mathrm{FCM}$ & 1D-Vector of FCM & PyTorch & CapsNet & Standard & $\begin{array}{l}\text { K-Means } \\
\text { Clustering }\end{array}$ & 10 & $\begin{array}{l}\text { Acc=71 } \\
\text { Sen=73 } \\
\text { Spec }=66\end{array}$ \\
\hline
\end{tabular}


TABLE II: Summary of papers published on rehabilitation of ASD patients using DL algorithms.

\begin{tabular}{|c|c|c|c|c|c|c|c|c|c|c|c|}
\hline Work & Datasets & $\begin{array}{c}\text { Type of } \\
\text { Applications }\end{array}$ & $\begin{array}{l}\text { Number } \\
\text { of Cases }\end{array}$ & Preprocessing & $\begin{array}{l}\text { Inputs } \\
\text { DNN }\end{array}$ & $\begin{array}{c}\text { DNN } \\
\text { Toolbox }\end{array}$ & DNNs & $\begin{array}{c}\text { Number of } \\
\text { Layers }\end{array}$ & Classifier & $\mathrm{K}$ fold & $\begin{array}{l}\text { Performance } \\
\text { Criteria (\%) }\end{array}$ \\
\hline \multirow{2}{*}{ 129] } & \multirow{2}{*}{ OSIE } & \multirow{2}{*}{-} & \multirow{2}{*}{$\begin{array}{l}20 \mathrm{ASD} \\
19 \mathrm{HC}\end{array}$} & \multirow{2}{*}{$\begin{array}{l}\text { HFM Construction, } \\
\text { Filtering Normalizing, DA }\end{array}$} & \multirow{2}{*}{$\begin{array}{l}\text { HFMs, Natural } \\
\text { Scene Images }\end{array}$} & Caffe & \multirow{2}{*}{ VGGNeT } & \multirow{2}{*}{50} & \multirow{2}{*}{ Softmax } & \multirow{2}{*}{13} & \multirow{2}{*}{$\begin{array}{l}\text { Acc=85 } \\
\text { Sen=80 } \\
\text { Spec }=89\end{array}$} \\
\hline & & & & & & TensorFlow & & & & & \\
\hline 731 & KDEF & Facial Expression Recognition & $\begin{array}{c}70 \\
\text { Individuals }\end{array}$ & DA & RGB Images $(562 \times 762)$ & Keras & DCNN & 44 & Softmax & $\mathrm{NA}$ & Acc $=78.32$ \\
\hline 1301 & $\begin{array}{l}\text { Clinical } \\
\text { Acquisition } \\
\end{array}$ & $\begin{array}{l}\text { Detecting Audio Regimes That Directly } \\
\text { Estimate ASD Severity Social Affect scores }\end{array}$ & 33 ASD & MFCC Spectrograms & 32 Spectrograms & $\mathrm{NA}$ & $\begin{array}{c}\text { Noisemes Network } \\
\begin{array}{c}\text { DiarTK Diarization } \\
\text { Network }\end{array} \\
\end{array}$ & $\begin{array}{l}\text { Standard } \\
\text { Network }\end{array}$ & $\begin{array}{c}\text { Synthetic } \\
\text { RF }\end{array}$ & $\mathrm{NA}$ & Acc $=84.7$ \\
\hline 721] & $\begin{array}{l}\text { Kaggle's } \\
\text { FER2013 } \\
\text { KDEF } \\
\end{array}$ & Facial Expression Recognition & $\mathrm{NA}$ & No & 48×48-Pixel Images & $\begin{array}{c}\text { Keras } \\
\text { (Tensorflow } \\
\text { Backend) }\end{array}$ & DCNN & 44 & Softmax & $\mathrm{NA}$ & Acc $=86.44$ \\
\hline [131] & SALICON & ASD Classification & $\begin{array}{l}14 \mathrm{ASD} \\
14 \mathrm{HC}\end{array}$ & $\begin{array}{l}\text { SalGAN Model, } \\
\text { Feature Extraction }\end{array}$ & Sequence of Image Patches & $\mathrm{NA}$ & SP-ASDNet & 11 & $\mathrm{NA}$ & $\mathrm{NA}$ & $\begin{array}{l}\text { Acc }=57.90 \\
\text { Rec=59.21 } \\
\text { Pre }=56.26\end{array}$ \\
\hline [132] & BigFaceX & Facial Expression Recognition & 196 Subjects & SW, Merge in the Channel Dimension, DA & $\begin{array}{l}\text { 5-channel Sub-Sequence Stacks } \\
\text { within a Specific Time Window }\end{array}$ & Keras & TimeConvNet & PreTrain Nets & Softmax & $\mathrm{NA}$ & Acc $=97.9$ \\
\hline 133] & $\begin{array}{l}\text { Different } \\
\text { Datasets }\end{array}$ & Suitable Courseware for Children with ASD & $\mathrm{NA}$ & $\begin{array}{l}\text { Interactive and Intelligent } \\
\text { Chatbot, NLP, Visual Aid }\end{array}$ & Different Inputs & $\mathrm{NA}$ & Different Nets & $\mathrm{NA}$ & $\mathrm{NA}$ & $\mathrm{NA}$ & $\mathrm{NA}$ \\
\hline \multirow{2}{*}{ [134] } & \multirow{2}{*}{ Camera Images } & \multirow{2}{*}{$\begin{array}{l}\text { Estimating Visual Attention in } \\
\text { Robot-Assisted Therapy }\end{array}$} & \multirow{2}{*}{6 ASD and ID } & $\begin{array}{l}\text { Resizing, Frame Extraction, } \\
\text { Visual Inspection }\end{array}$ & \multirow{2}{*}{$\begin{array}{l}5 \text { Facial Landmarks - } 36 \\
\text { HOG Descriptors }\end{array}$} & \multirow{2}{*}{$\mathrm{NA}$} & R-CNN & VGG-16 & K-NN & \multirow{2}{*}{10} & \multirow{2}{*}{$\begin{array}{l}\text { Acc }=88.2 \\
\text { Pre } 83.3 \\
\text { Sens }=83.0 \\
\text { Spec }=87.3\end{array}$} \\
\hline & & & & $\begin{array}{c}\text { Face Detection (Viola-Jones), } \\
\text { Feature Extraction (HOG Descriptors) }\end{array}$ & & & MTCNN & $\begin{array}{l}\text { Cascaded CNNs } \\
\text { Architecture }\end{array}$ & Naive & & \\
\hline 135] & $\begin{array}{c}\text { Sensor } \\
\text { Data }\end{array}$ & Automatic SMM detection & $\begin{array}{l}6 \mathrm{ASD} \\
5 \mathrm{HC}\end{array}$ & Resampling, Filtering, SW & Time-Series of Multiple Sensors & Keras & CNN-LSTM & 13 & $\begin{array}{c}\text { Majority } \\
\text { Voting }\end{array}$ & $\mathrm{NA}$ & $\mathrm{NA}$ \\
\hline 136] & КОМАA & Facial Expression Recognition & 55 subjects & $\begin{array}{l}\text { Segmentation, Different } \\
\text { Features, Z-scores }\end{array}$ & Greedy Forward Feature Selection & $\mathrm{NA}$ & $\mathrm{CNN}$ & 9 & SVM & $\mathrm{NA}$ & Acc $=96$ \\
\hline [137] & $\begin{array}{c}\text { Story-Telling } \\
\text { Narrative Corpora }\end{array}$ & ASD Classification & $\begin{array}{l}31 \mathrm{ASD} \\
36 \mathrm{HC}\end{array}$ & DA, ChineseWord2Vec & 32-Dimensional Word Vector & $\mathrm{NA}$ & LSTM & 1 & $\begin{array}{l}\text { Coherence Representation of } \\
\text { LSTM Forget Gate }\end{array}$ & $\mathrm{NA}$ & Acc $=92$ \\
\hline 138 & $\begin{array}{l}\text { Ext-Dataset } \\
\text { (video dataset) }\end{array}$ & ASD Classification using Eye Tracking & $\begin{array}{l}136 \mathrm{ASD} \\
136 \mathrm{HC}\end{array}$ & $\begin{array}{l}\text { TLD Method, Accumulative } \\
\text { Histogram Computation }\end{array}$ & $\begin{array}{c}\text { Angle Histogram, Length } \\
\text { Histogram and Fused Histogram, }\end{array}$ & Keras & LSTM & 4 & $\mathrm{NA}$ & 10 & $\begin{array}{l}\text { Acc=92.6 } \\
\text { Sen=1.9 } \\
\text { Spec=93.4 }\end{array}$ \\
\hline [139] & MIT1003 & $\begin{array}{l}\text { Predicting Visual Attention } \\
\text { of Children with ASD. }\end{array}$ & 300 Images & $\mathrm{NA}$ & Raw Images & $\mathrm{NA}$ & DCN & 26 & $\mathrm{NA}$ & $\mathrm{NA}$ & $\begin{array}{c}\text { SII }=67.8 \\
\text { CC }=76.9 \\
\text { AUC-J }=83.4\end{array}$ \\
\hline 751] & $\begin{array}{l}\text { Scan Path Data, } \\
\text { Including Location } \\
\text { and Duration }\end{array}$ & ASD Classification & $\begin{array}{l}14 \mathrm{ASD} \\
14 \mathrm{HC}\end{array}$ & DA Methods & Image, Data Points & Pytorch & ResNet18 & Standard & Softmax & $\mathrm{NA}$ & $\begin{array}{l}\text { Acc=55.13 } \\
\text { Sen=63.5 } \\
\text { Spec= }=47.1\end{array}$ \\
\hline [140] & $\begin{array}{l}\text { UCI Machine } \\
\text { Learning } \\
\text { Repository }\end{array}$ & ASD Classification & 704 & Different Methods & Preprocessed Data & $\mathrm{NA}$ & $\mathrm{CNN}$ & 7 & $\mathrm{NA}$ & $\mathrm{NA}$ & $\begin{array}{l}\text { Acc }=99.53 \\
\text { Sens }=99.39 \\
\text { Spec }=100\end{array}$ \\
\hline [76] & $\begin{array}{l}\text { Eye Tracking } \\
\text { Scanpath }\end{array}$ & ASD Classification & $\begin{array}{l}29 \mathrm{ASD} \\
30 \mathrm{HC}\end{array}$ & $\begin{array}{l}\text { Visualization of Eye-Tracking Scanpaths } \\
\text { Scaling Down, PCA }\end{array}$ & $100 * 100$ Image & $\begin{array}{l}\text { Keras, } \\
\text { Scikit- } \\
\text { Learn }\end{array}$ & $\mathrm{AE}$ & 8 & $\begin{array}{l}\text { K-Means } \\
\text { Clustering }\end{array}$ & $\mathrm{NA}$ & $\begin{array}{l}\text { Silhouette } \\
\text { score }=60\end{array}$ \\
\hline 141] & Video Data & $\begin{array}{c}\text { Engagement Estimation } \\
\text { of Children with ASD During a } \\
\text { Robot-Assisted Autism Therapy }\end{array}$ & 30 children & $\mathrm{NA}$ & $\begin{array}{l}\text { Cropped Face Images } \\
\quad(256 * 256)\end{array}$ & $\begin{array}{c}\text { Keras } \\
\text { with } \\
\text { Tensorflow } \\
\text { Backend }\end{array}$ & CultureNet & $\begin{array}{l}\text { R-CNN + } \\
\text { ResNet50+ } \\
\text { 5FC layers }\end{array}$ & Softmax & $\mathrm{NA}$ & $\begin{array}{l}\text { ICC }=43.35 \\
\text { CCC }=43.18 \\
\text { PC }=45.17\end{array}$ \\
\hline [142] & $\begin{array}{l}\text { YouTube } \\
\text { ASD } \\
\text { Dataset }\end{array}$ & $\begin{array}{l}\text { Modeling Typical and Atypical Behaviors } \\
\text { in ASD Children }\end{array}$ & 68 video Clips & Different Methods & $\begin{array}{l}\text { Sequences of Individual Frames } \\
\text { at a Rate of } 30 \mathrm{fps}\end{array}$ & $\begin{array}{l}\text { openCV, } \\
\text { Caffe }\end{array}$ & DCNN & $\mathrm{NA}$ & DT & 5 & $\begin{array}{l}\text { Avg Pre=73 } \\
\text { Avg Recall=75 } \\
\text { Avg Acc } 71\end{array}$ \\
\hline 143] & $\begin{array}{l}\text { Video } \\
\text { Dataset }\end{array}$ & $\begin{array}{l}\text { Behavioral Data Extracted } \\
\text { from Video Analysis of } \\
\text { Child-Robot Interactions. }\end{array}$ & $\begin{array}{l}5 \mathrm{ASD} \\
7 \mathrm{HC}\end{array}$ & $\begin{array}{l}\text { Segmentation, Upper Body tracking, } \\
\text { Laban Movement Analysis to } \\
\text { Drive Weight, Different features }\end{array}$ & $\begin{array}{l}3 \text { Movement Features with } 68 \\
\text { Facial Key-Points }\end{array}$ & $\mathrm{NA}$ & $\mathrm{CNN}$ & 10 & Softmax & $\mathrm{NA}$ & $\begin{array}{l}\text { Acc=88.46 } \\
\text { Pre=89.12 } \\
\text { Recall }=88.53\end{array}$ \\
\hline 144] & $\begin{array}{c}\text { Video } \\
\text { Dataset }\end{array}$ & $\begin{array}{l}\text { Developing Automatic SMM } \\
\text { Detection Systems }\end{array}$ & $6 \mathrm{ASD}$ & $\begin{array}{l}\text { Resampling, Filtering, SW, } \\
\text { Data Balancing, Normalizing }\end{array}$ & $\begin{array}{l}\text { Time-Series of Multiple } \\
\text { Accelerometer Sensors }\end{array}$ & $\begin{array}{l}\text { Deeppy } \\
\text { Library }\end{array}$ & $\mathrm{CNN}$ & 8 & svM & $\mathrm{NA}$ & F1-score $=95$ \\
\hline [145] & ASD Screening & Autism Screening & $\begin{array}{l}513 \mathrm{ASD} \\
189 \mathrm{HC}\end{array}$ & $\begin{array}{l}\text { Cleaning Missing Values and Outliers, } \\
\text { Visualization, Identity Mapping }\end{array}$ & $\begin{array}{l}\text { The Embedded Categorical Variables } \\
\text { are Concatenated with Numerical } \\
\text { Features as New Feature Vectors }\end{array}$ & $\mathrm{NA}$ & DENN & 4 & Sigmoid & $\mathrm{NA}$ & $\begin{array}{c}\text { Acc }=100 \\
\text { Spec }=99 \\
\text { Sen }=100 \\
\text { F1-score }=99\end{array}$ \\
\hline 146] & $\begin{array}{l}\text { ASD Screening } \\
\text { Datasets }\end{array}$ & $\begin{array}{l}\text { Classification of } \\
\text { Adults with ASD } \\
\end{array}$ & - & $\begin{array}{l}\text { Handling of Missing Values, Variable } \\
\text { Reduction, Normalization, and } \\
\text { Label Encoding }\end{array}$ & Normalized Variables & Keras & DNN & 7 & Sigmoid & $\mathrm{NA}$ & $\begin{array}{l}\text { Acc }=99.40 \\
\text { Sen=97.89 } \\
\text { Spec }=100 \\
\end{array}$ \\
\hline
\end{tabular}




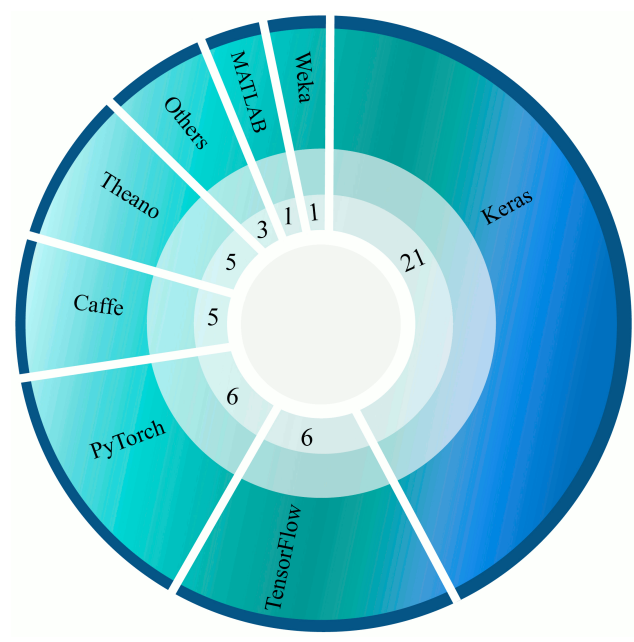

Fig. 13: Number of DL tools used for the diagnosis and rehabilitation of ASD patients in reviewed papers.

\section{DISCUSSION}

In this study, we performed a comprehensive overview of the investigations conducted in the scope of ASD diagnostic CAD systems as well as DL-based rehabilitation tools for ASD patients. In the field of ASD diagnosis, numerous papers have been published using functional and structural neuroimaging data as well as rehabilitation tools, as summarized in Table III in the appendix. A variety of DL toolboxes have been proposed for implementing deep networks. In Tables $\mathbb{I}$ and III the types of DL toolboxes utilized for each study are depicted, and the total number of their usage is demonstrated in Figure 13. The Keras toolbox is used in the majority of the studies due to its simplicity. Keras offers a consistent highlevel application programming interface (APIs) to build the models more straightforward, and by using powerful backends such as TensorFlow, its performance is sound. Additionally, due to all pre-trained models and available codes on platforms such as GitHub, Keras is quite popular among researchers.

Number of DL networks used for the ASD detection in the reviewed works is shown in Figure 14 Among the various DL architectures, CNN is found to be the most popular one as it has achieved more promising results compared to other deep methodologies. The autoencoder, as well as RNN, have yielded favorable results. It can be noted that in recent years, the number of DL-based papers has increased exponentially due to their sound performance and also the availability of vast and thorough datasets.

The number of various classification algorithms used in DL networks are shown in Figure 15. One of the best and most widely used is the Softmax algorithm (Tables $\Pi$ and $\Pi$ ). It is most popular since it is differentiable in the entire domain and computationally less expensive.

\section{Challenges}

Some of the most substantial challenges in ASD diagnosis scope using DL-based techniques are addressed in this section, which comprise database and algorithmic problems. There are only two-class brain structural and functional datasets (ASD

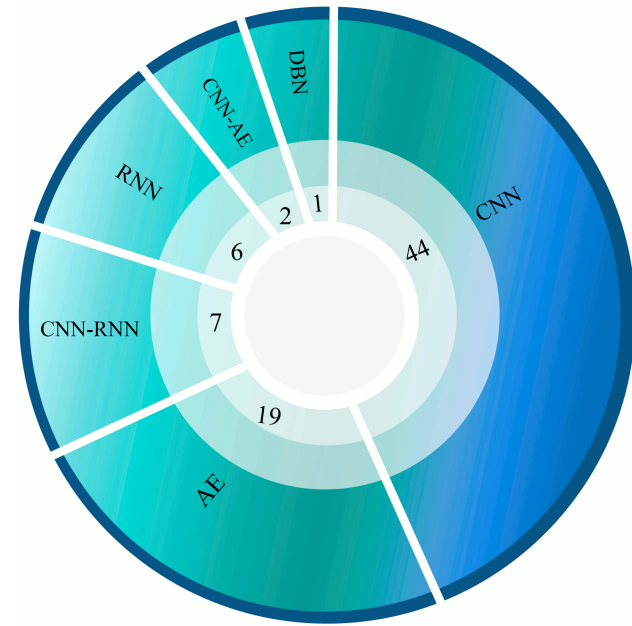

Fig. 14: Number of of DL networks used for ASD detection and rehabilitation in the reviewed works.

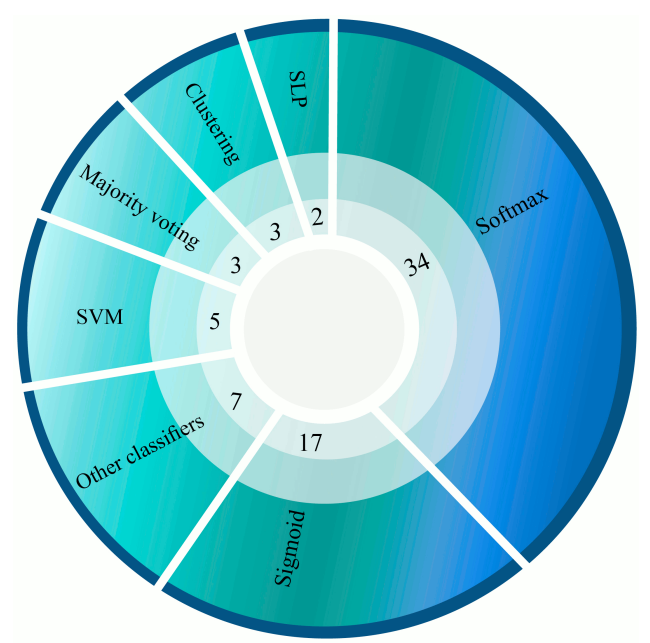

Fig. 15: Number of various classification algorithms used for the detection of ASD and rehabilitation in DL.

and healthy) available in the public domain. Hence, researchers are not able to broaden their investigation to all sub-types of ASD. Two of the cheapest and most pragmatic functional neuro-screening modalities for diagnosis of ASD are EEG, and fNIRS. But unfortunately the deficiency of freely available datasets has resulted in little research in this area. Another obstacle is that multi-modality databases such as EEG-fMRI are not available to researchers to evaluate the effectiveness of incorporating information of different imaging modalities to detect ASD. Although fMRI and sMRI data are ubiquitous in the ABIDE dataset, the results of merging these structural and functional data for ASD diagnosis using DL have not yet been investigated. Another problem grappling the researchers is designing the DL-based rehabilitation systems with hardware resources. Nowadays, assistive tools such as Google Colab are available to researchers to improve the processing power; however, the problems still prevail when implementing these systems in real-world scenarios. 


\section{CONCLUSION AND FUTURE WORKS}

ASD is typically characterized by social disorders, communication deficits, and stereotypical behaviors. Numerous computer-aided diagnosis systems and rehabilitation tools have been developed to assist patients with autism disorders. In this survey, research on ASD diagnosis applying DL and functional and structural neuroimaging data were first assessed. The researchers have taken advantage of deep CNNs, RNNs, AEs, and CNN-RNN networks to improve the performance of their systems. Boosting the accuracy of the system, the capability of generalizing and adapting to differing data and real-world challenges, as well as reducing the hardware power requirements to the extent that the final system can be utilized by all are the principal challenges of these systems. To enhance the accuracy and performance of CADS for ASD detection in the future, deep reinforcement networks (RL) or GANs can be exploited. Scarcity of data is always an aparamount problem in the medical field that can be resolved relatively with the help of these deep GANs. Also, as another direction for future works, handcrafted features can be extracted from data and fed to DL networks in addition to raw data; this can help increasing performance by adding the potential of traditional methods to DL-based models.

Many researchers have proposed various DL-based rehabilitation tools to aid the ASD patients. Designing a reliable, accurate, and wearable low power consumption DL algorithm based device is the future tool for ASD patients. An achievable rehabilitation tool is to wear smart glasses to help the children with ASD. These glasses with the built-in cameras will acquire the images from the different directions of environment. Then the DL algorithm processes these images and produces meaningful images for the ASD children to better communicate with their surroundings.

\section{APPENDIX A}

\section{STATISTICAL METRICS}

This section demonstrates the equations for the calculation of each evaluation metric. In these equations, True positive (TP) is the correct classification of the positive class, True negative (TN) is the correct classification of the negative class, False positive (FP) is the incorrect prediction of the positives, False negative (FN) is the incorrect prediction of the negatives.

$$
\begin{gathered}
\text { Accuracy }(\text { Acc })=\frac{T P+T N}{T P+T N+F P+F N} \\
\text { Specificity }(\text { Spec })=\frac{T N}{T N+F P} \\
\text { Sensitivity }(\text { Sen })=\frac{T P}{T P+F N} \\
\text { Precision }(\text { Prec })=\frac{T P}{T P+F P} \\
F 1-\text { Score }=2 * \frac{\text { Prec } * \text { Sens }}{\text { Prec }+ \text { Sens }}
\end{gathered}
$$

The receiver operating characteristic curve (ROC-curve) depicts the performance of the proposed model at all classification thresholds. It is the graph of true positive rate vs. false positive rate (TPR vs. FPR). Equations for calculation of TPR and FPR are presented below.

$$
\begin{aligned}
& T P R=\frac{T P}{T P+F N} \\
& F P R=\frac{F P}{F P+T N}
\end{aligned}
$$

AREA UNDER THE ROC CURVE (AUC)

AUC presents the area under the ROC-curve from $(0,0)$ to $(1,1)$. It provides the aggregate measure of all possible classification thresholds. AUC has a range from 0 to 1 . A $100 \%$ wrong classification will have AUC value of 0.0 , while a $100 \%$ correct classified version will have the AUC value of 1.0. It has two folded advantages. One is that it is scaleinvariant, which implies how well the model is predicted rather than checking the absolute values. The second advantage is that it is classification threshold-invariant as it will verify the performance of the model irrespective of the threshold being selected.

\section{APPENDIX B}

Table III shows details of Deep Nets in all the papers reviewed in this study.

\section{ACKNOWLEDGMENT}

$\mathrm{MB}$ is supported by a NHMRC Senior Principal Research Fellowship (1059660 and 1156072). MB has received Grant/Research Support from the NIH, Cooperative Research Centre, Simons Autism Foundation, Cancer Council of Victoria, Stanley Medical Research Foundation, Medical Benefits Fund, National Health and Medical Research Council, Medical Research Futures Fund, Beyond Blue, Rotary Health, A2 milk company, Meat and Livestock Board, Woolworths, Avant and the Harry Windsor Foundation, has been a speaker for Astra Zeneca, Lundbeck, Merck, Pfizer, and served as a consultant to Allergan, Astra Zeneca, Bioadvantex, Bionomics, Collaborative Medicinal Development, Lundbeck Merck, Pfizer and Servier - all unrelated to this work. 
TABLE III: Details of Deep Nets. For ASD diagnosis and Rehabilitation.

\begin{tabular}{|c|c|c|c|c|c|c|}
\hline Work & Network & Details for Deep Networks & Dropout & Classifier & Optimizer & Loss Function \\
\hline 24 & 2CC3D & CNN Layers (6) + Pooling Layers (4) + FC Layers (2) & $\frac{2(\text { rate }=0.5)}{2(\text { rate }=0.65)}$ & Sigmoid & $\mathrm{NA}$ & $\mathrm{BCE}$ \\
\hline [77] & $2 \mathrm{CC} 3 \mathrm{D}$ & CNN Layers (6) + Pooling Layers (4) + FC Layers (3) & $\mathrm{NA}$ & Sigmoid & $\mathrm{NA}$ & $\mathrm{NA}$ \\
\hline - 78 & 3D-CNN & CNN Layers (2) + LReLU Actication + Pooling Layers (1) + FC Layers (1) & 3 (rate=NA) & Softmax & SGD & MNLL \\
\hline$[59]$ & LSTM & LSTM Layers (1) + Pooling Layers (1) + FC Layers (3) & 1 (rate $=0.5)$ & Sigmoid & Adadelta & MSE \\
\hline 79 & $\mathrm{CNN}$ & CNN Layers (2) + ReLU Activation + BN Layers (4) + FC Layers (3) & $\begin{array}{l}\text { (rate }=0.3 \text { ) } \\
2 \text { (rate }=0.7 \text { ) }\end{array}$ & Softmax & Adam & $\mathrm{NA}$ \\
\hline 80 & $2 \mathrm{CC} 3 \mathrm{D}$ & CNN Layers (6) + Pooling Layers (4) + FC Layers (2) & 2 (rate=NA) & Sigmoid & $\mathrm{NA}$ & $\mathrm{NA}$ \\
\hline-81$]^{-}$ & $\mathrm{CNN}$ & CNN Layers (2) + ELU Activation + Pooling Layers (2) + FC Layers (2) & $\mathrm{NA}$ & Sigmoid & SGD & $\mathrm{NA}$ \\
\hline 82 & $\mathrm{AE}$ & Standard AE with Tanh Actication & NA & SLP & $\mathrm{NA}$ & $\begin{array}{ll}\text { MSE } \\
\text { BCE }\end{array}$ \\
\hline 83$]$ & G-CNN & Proposed G-CNN with 3 Layer CNN & (rate $=0.3)$ & Softmax & Adam & NA \\
\hline 84 & $\begin{array}{l}\text { BrainNetCNN with } \\
\text { Proposed Layers }\end{array}$ & $\begin{array}{c}\text { Element-wise layer (1) + E2E layers (2) + E2N layer (1) + N2G layer (1) } \\
\text { + FC layers (3)+ Leaky ReLU Activation+ Tanh Activation }\end{array}$ & $\begin{array}{c}\text { (rate }=0.5) \\
1(\text { rate }=0.6)\end{array}$ & Softmax & Adam & Proposed Loss Function \\
\hline 85 & DAE & Standard DAE & $\mathrm{NA}$ & $\mathrm{NA}$ & $\mathrm{NA}$ & Proposed Loss function \\
\hline 86 & LeNet-5 & Standard LeNet-5 Architecture & $\mathrm{NA}$ & Softmax & $\mathrm{NA}$ & NA \\
\hline 87 & SAE & SAE with LSF Activation & $\mathrm{NA}$ & Softmax & LBFGS & $\mathrm{NA}$ \\
\hline 62 & MCNNEs & CNN Layers (3) + ReLU Activation + Pooling Layers (3) + FC Layers (1) & $1($ rate $=0.5)$ & Binary SR & \begin{tabular}{c|c|c|} 
Adam \\
Adamax
\end{tabular} & BCE \\
\hline 88 & 3D-CNN & CNN Layers (2) + ELU Activation + Pooling Layers (2) + FC Layers (3) & NA & Sigmoid & SGD & $\mathrm{BCE}$ \\
\hline & 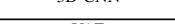 & 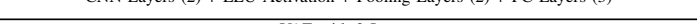 & 102 & ighiorid & Adam & MSD \\
\hline 89 & VAE & VAE with 3 Layers & $\mathrm{NA}$ & NA & Adadelta & Proposed Loss Function \\
\hline 90 & LSTM & LSTM Layers (1) + Pooling Layers (1) + FC Layers (1) & 1 (rate $=0.5$ ) & Sigmoid & Adadelta & $\mathrm{BCE}$ \\
\hline 91 & SAE & SAE Layers (3) + Sigmoid Activation & NA & Clustering & Proposed Opt. & $\mathrm{NA}$ \\
\hline 92 & SAE & SAE Layers $(8)+$ Sigmoid Activation & $\mathrm{NA}$ & SR & L-BFGS & MSE \\
\hline 93 & LSTM & LSTM Layers (2) + Pooling Layers (1) + FC Layers (2) & NA & Sigmoid & Adam & $\begin{array}{ll}\text { BCE } \\
\text { MSE }\end{array}$ \\
\hline 94 & Multichannel DANN & $\begin{array}{l}\text { 3 MLP (1 Dropout Layer and } 4 \text { Dense Layers) + Self-Attention (3) + Fusion (3) } \\
\text { + Aggregation Layer + Dense Layer (1) + ReLU, ELU, Tanh Activations }\end{array}$ & 1 (rate=NA) & Sigmoid & $\mathrm{NA}$ & $\mathrm{CE}$ \\
\hline 95 & SSAE & 3 SSAE Layers & NA & Softmax & $\begin{array}{l}\text { Scaled Conjugate } \\
\text { Gradient Descent }\end{array}$ & Proposed Loss Function \\
\hline 96 & 1D-CNN & CNN Layers (1) + Pooling Layers (1) + FC Layers (1) & $($ rate $=0.2)$ & Softmax & Adam & $\mathrm{NA}$ \\
\hline - 97 & $\mathrm{CNN}$ & CNN Layers (6) + Pooling Layers (4) + BN Layers (2) + FC Layers (2) & 1 (rate $=0.25)$ & Sigmoid & Adam & Propose Loss Function \\
\hline-98 & 1D CAE-CNN & Encoder (4 layers) + Decoder (4 layers) + CNN layers (2) + pooling layers (2) + FC layers (2) & $\mathrm{NA}$ & NA & $\mathrm{NA}$ & $\mathrm{NA}$ \\
\hline 99 & AlexNet & Standard AlexNet Architecture & $\mathrm{NA}$ & Softmax & $\mathrm{NA}$ & $\mathrm{CE}$ \\
\hline$[100]$ & ASD-DiagNet & Proposed DiagNet & $\mathrm{NA}$ & SLP & $\mathrm{NA}$ & NA \\
\hline 60 & Auto-ASD-Network & Proposed Auto-ASD-Network & $\mathrm{NA}$ & SVM & $\mathrm{NA}$ & NLLF \\
\hline 61 & $\mathrm{CNN}$ & CNN layers (7) + Pooling layers (7) + FC layers (3) & 1 (rate $=0.25$ ) & MLP & $\mathrm{NA}$ & NA \\
\hline 101$]$ & 2 SdAE-CNN & Proposed SDAE-CNN with 7 Layes CNN & NA & Softmax & NA & $\mathrm{NA}$ \\
\hline 102 & 3D-FCNN & CNN Layers (9) + PReLU Activation + FC Layers (3) & $\mathrm{NA}$ & Softmax & SGD & $\mathrm{CE}$ \\
\hline 103 & SSAE & 2 Layers SSAE & $\mathrm{NA}$ & Softmax & NA & $\mathrm{NA}$ \\
\hline 104 & 3D-CNN & CNN layers (7) + Pooling Layers (3) + FC Layers (2) + Log-Likelihood Activation & 2 (rate $=0.2)$ & $\mathrm{NA}$ & SGD & MNLL \\
\hline 105 & $\mathrm{GCN}$ & GCN with ReLU and Sigmoid Actication & $($ rate $=0.3)$ & Softmax & NA & $\begin{array}{ll}\mathrm{CE} \\
\mathrm{MSE}\end{array}$ \\
\hline & $\mathrm{AE}$ & SAE wth Tanh Activation & (Twe & Ботитол & 12 & $\begin{array}{c}\text { MSE } \\
\mathrm{BCE}\end{array}$ \\
\hline 106 & LSTM & Proposed Deep Nework & (rate $=0.5)$ & Sigmoid & Adadelta & $\begin{array}{l}\text { BCE } \\
\text { MSE }\end{array}$ \\
\hline$[107$ & 2 SdAE-MLP & Proposed 2-SDAE-MLP Network & $\mathrm{NA}$ & Softmax & $\mathrm{NA}$ & MSE \\
\hline 108 & 3D-CNN & CNN Layers (2) + ReLU Activation + Pooling Layers (2) + FC Layers (2) & $\mathrm{NA}$ & Sigmoid & SGD & $\mathrm{BCE}$ \\
\hline 109 & DBN & DBN with 5 Hidden Layers & $\mathrm{NA}$ & LR & NA & NA \\
\hline 110 & FeedFWD & Dense layers (5) + LReLU activation & $3($ rate $=\mathrm{NA})$ & NA & Adam & $\mathrm{BCE}$ \\
\hline 111 & $\begin{array}{l}\text { Ensemble } \\
\text { of } 5 \text { SAEs } \\
\text { and MLPs }\end{array}$ & $5[\mathrm{AE}(3)+\mathrm{MLP}(2)]+$ Softmax & $5($ rate $=\mathrm{NA})$ & $\begin{array}{l}\text { Averaging the } \\
\text { Softmax } \\
\text { Activation } \\
\text { Probabilities }\end{array}$ & $\mathrm{NA}$ & $\mathrm{NA}$ \\
\hline$[112]$ & Multi-Channel CNN & CNN Layers (5) + ReLU Activation + Pooling Layers (2) + FC Layers (5) & $\mathrm{NA}$ & Softmax & $\mathrm{NA}$ & $\mathrm{CE}$ \\
\hline 63 & 34 SAEs & 34 [ SAE network (2)] & $\mathrm{NA}$ & PSVM & L-BFGS & $\mathrm{NA}$ \\
\hline 113 & DDUNET & Proposed DDUNET with 11 blocks and ReLU Activation & $($ rate $=0.1)$ & NA & SGD & $\mathrm{CE}$ \\
\hline 114 & SNCAE & Proposed SNCAE Newtork & NA & Softmax & $\mathrm{NA}$ & $\mathrm{NA}$ \\
\hline 115 & SpAE & SpAE with 2 Networks & $\mathrm{NA}$ & Softmax & $\mathrm{NA}$ & MSE \\
\hline 116 & DAE & $\mathrm{AE}(3)+\mathrm{SELU}$ Activation & $\mathrm{NA}$ & NA & Adam & Sum of MSE $+2 \mathrm{CE}+\mathrm{CC}$ \\
\hline 117 & DCNN & CNN Layers (6) + ReLU Activation + Pooling Layers (6) + FC Layers (4) & $\mathrm{NA}$ & Sigmoid & Adam & $\mathrm{BCE}$ \\
\hline 118 & FastSurfer CNN & Proposed FastSurfer CNN Network & $\mathrm{NA}$ & Softmax & Adam & Logistic \& Dice Losses \\
\hline 119 & 3D-CNN & CNN Layers (3) + ReLU Activation + Pooling Layers (3) + FC Layers (2) & 2 (rate $=0.5)$ & Softmax & Adadelta & $\mathrm{CE}$ \\
\hline 120 & 3D-UNET & DCNN Layers (7) + ReLU Activation + Pooling Layers (2) + BN Layers (6) & 2 (rate $=0.5)$ & Softmax & SGD & weighted $\mathrm{CE}$ \\
\hline 121 & CNN-GRU & CNN Layers (4) + GRU Layers (2) + ReLU Activation + Pooling Layers (2) + FC Layers (5) & NA & Sigmoid & Adam & $\mathrm{BCE}$ \\
\hline 122 & 1D CNN - LSTM & $\begin{array}{l}\text { Proposed 1D-CNN LSTM with ReLU Activation } \\
\end{array}$ & $($ rate $=0.2)$ & Softmax & Adam & $\mathrm{CCE}$ \\
\hline 123 & CGRNN & $\begin{array}{l}\text { CNN layers (3) + ReLU Activation + Pooling Layers (1) } \\
\text { + GRU Layers (1) + Sigmoid Activation + FC Layer (1) }\end{array}$ & $1($ rate $=0.5)$ & NA & Adam & BCE \\
\hline$[124$ & ConvNet & Variation of the U-net Convolutional Architecture & $\mathrm{NA}$ & NA & Adam & Proposed Loss Function \\
\hline 125 & 3D-CNN & CNN Layers (2) + ELU Activations + Pooling Layers (2) + FC Layers (2) & $\mathrm{NA}$ & Sigmoid & SGD & $\mathrm{BCE}$ \\
\hline 126 & 3DCNN C-LSTM & $\begin{array}{c}\text { CNN Layers (8) + Conv-Bi LSTM Layers (2) + Sigmoid Activation (for LSTM) } \\
\text { + Pooling Layers (1) + FC Layers (1) }\end{array}$ & $8($ rate $=0.2)$ & Softmax & Adam & $\mathrm{CE}$ \\
\hline$[127$ & $\mathrm{AE}$ & Proposed AE with 7 Layers & $\mathrm{NA}$ & DNN & $\mathrm{NA}$ & $\mathrm{NA}$ \\
\hline 128 & CapsNets & Standard Architecture & $\mathrm{NA}$ & K-Means Clustering & Adam & Proposed Loss Function \\
\hline 129 & VGGNets + ASDNet & CNN Layers (27) + ReLU Activation + Pooling Layers (10) + FC Layers (6) & $6($ rate $=0.5)$ & Softmax & SGD & $\mathrm{CE}$ \\
\hline 73 & DCNN & CNN Layers (7) + Activation+ Pooling Layers (13) + FC Layers (3) + BN Layers (10) & \begin{tabular}{c|c}
7 (rate $=0.25)$ \\
3 (rate $=0.5)$ \\
\end{tabular} & Softmax & SGD & $\mathrm{NA}$ \\
\hline 130 & $\begin{array}{c}\text { Noisemes net } \\
\text { DiarTK Diarization net }\end{array}$ & Standard Networks & NA & RF & $\mathrm{NA}$ & NA \\
\hline 72 & DCNN & CNN Layers (7) + ELU Activation + Pooling Layers (13) + FC Layers (3) + BN Layers (10) & $\frac{7 \text { (rate }=0.25)}{3 \text { (rate }=0.5)}$ & Softmax & SGD & NA \\
\hline 131 & SP-ASDNet & CNN Layers (2) + LSTM Layers (2) + Pooling Layers (3) + FC Layers (2) & $2($ rate $=\mathrm{NA})$ & $\mathrm{NA}$ & Adam & BCE \\
\hline 132 & TimeConvNet & $\begin{array}{l}\text { Convolutional Spatiotemporal Encoding Layer+ Backbone Convolutional } \\
\text { Neural Network Architecture (Mini-Xception, ResNet20, MobileNetV2) }\end{array}$ & NA & Softmax & Adam & $\mathrm{CCE}$ \\
\hline$[133$ & Different Networks & Proposed Structure & $\mathrm{NA}$ & $\mathrm{NA}$ & $\mathrm{NA}$ & $\mathrm{NA}$ \\
\hline 134 & RCNN & $\begin{array}{c}\text { VGG-16 } \\
\text { Cascaded CNNS Architecture }\end{array}$ & NA & KNN & NA & NA \\
\hline 135 & $\frac{\text { MTCNN }}{\text { CNN-LSTM }}$ & $\begin{array}{c}\text { Cascaded CNNs Architecture } \\
\text { CNN Layers (3) + LSTM Layers (1) + ReLU Activation } \\
\text { + Pooling Layers (3) + FC Layers (3) }\end{array}$ & $\begin{array}{l}1 \text { (rate }=0.5) \\
1 \text { (rate }=0.2)\end{array}$ & Softmax & SGD & NA \\
\hline$[136$ & $\mathrm{CNN}$ & CNN Layers (4) + Pooling Layers (2) + FC Layers (2) & NA & Softmax & $\mathrm{NA}$ & $\mathrm{NA}$ \\
\hline 137 & LSTM & LSTM layer (1) & $\mathrm{NA}$ & Coherence Representation & $\mathrm{NA}$ & $\mathrm{NA}$ \\
\hline 138 & LSTM & LSTM Layers (3) + Sigmoid Activation + FC Layers (1) & $\mathrm{NA}$ & $\mathrm{NA}$ & $\mathrm{NA}$ & $\mathrm{CE}$ \\
\hline$[139]$ & DCN & CNN Layers (17) + Pooling Layers (3) + Deconvolution Layers (3) + Learned Priors (3) & $\mathrm{NA}$ & NA & $\mathrm{NA}$ & Proposed Loss Function \\
\hline 75 & Pretrained Resnet 18 & Standard ResNet-18 Architecture & Standard & Standard & Adam & BCE \\
\hline$[140]$ & $\mathrm{CNN}$ & CNN Layers (2) + ReLU Activation + Pooling Layers (2) + FC Layers (2) & 1 (rate $=0.5)$ & NA & Adam & BCE \\
\hline 76 & $\mathrm{AE}$ & AE with 8 Layers & NA & K-Means Clustering & NA & NA \\
\hline 141 & CultureNet & Faster R-CNN + Modified ResNet50 + 5FC Layers & $\mathrm{NA}$ & Softmax & Adelta & Proposed Loss Function \\
\hline 142 & DCNN & Proposed DCNN Architecture with Different Layers & NA & Decision Tree (DT) & Manual Optimization & $\mathrm{NA}$ \\
\hline 143 & $\mathrm{CNN}$ & CNN Layers (2) + ReLU Activation + FC Layers (3) & 4 (rate $=0.2)$ & Softmax & $\mathrm{NA}$ & $\mathrm{NA}$ \\
\hline 144 & $\begin{array}{l}\text { SA-B3D with } \\
\text { LSTM Model }\end{array}$ & CNN Layers (5) + LSTM Layers (1) + Pooling Layers (4) + FC Layers (1) & NA & Sigmoid & Adam & $\begin{array}{c}\text { CE } \\
\text { Proposed Loss Function }\end{array}$ \\
\hline 74 & $\mathrm{CNN}$ & CNN Layers (3) + ReLU Activation + Pooling Layers (3) + FC Layers (1) & $\mathrm{NA}$ & SVM & SGD & NA \\
\hline 145 & DENN & Proposed DENN Architecture with ReLU Activation + FC Layers (2) & $\mathrm{NA}$ & Sigmoid & mini-batch SGD & $\mathrm{CCE}$ \\
\hline 146 & DNN & Propoded DNN with ReLU Activation + FC Layers (2) & $\begin{array}{l}\text { (rate }=0.2) \\
\text { (rate }=0.4)\end{array}$ & Sigmoid & Adam & $\mathrm{BCE}$ \\
\hline
\end{tabular}




\section{REFERENCES}

[1] J. Kang, X. Han, J. Song, Z. Niu, and X. Li, "The identification of children with autism spectrum disorder by svm approach on eeg and eye-tracking data," Computers in Biology and Medicine, p. 103722. 2020.

[2] B. S. Abrahams and D. H. Geschwind, "Advances in autism genetics: on the threshold of a new neurobiology," Nature reviews genetics, vol. 9, no. 5, pp. 341-355, 2008.

[3] E. Stevens, D. R. Dixon, M. N. Novack, D. Granpeesheh, T. Smith, and E. Linstead, "Identification and analysis of behavioral phenotypes in autism spectrum disorder via unsupervised machine learning," International journal of medical informatics, vol. 129, pp. 29-36, 2019.

[4] M. J. Maenner, K. A. Shaw, J. Baio et al., "Prevalence of autism spectrum disorder among children aged 8 years-autism and developmental disabilities monitoring network, 11 sites, united states, 2016," MMWR Surveillance Summaries, vol. 69, no. 4, p. 1, 2020.

[5] S. Yazdani, A. Capuano, M. Ghaziuddin, and C. Colombi, "Exclusion criteria used in early behavioral intervention studies for young children with autism spectrum disorder," Brain Sciences, vol. 10, no. 2, p. 99, 2020.

[6] C. Andy and R. S. Masters, "Improving motor skill acquisition through analogy in children with autism spectrum disorders," Psychology of Sport and Exercise, vol. 41, pp. 63-69, 2019.

[7] S. Mostafa, L. Tang, and F.-X. Wu, "Diagnosis of autism spectrum disorder based on eigenvalues of brain networks," IEEE Access, vol. 7, pp. 128 474-128486, 2019.

[8] A. Kazeminejad and R. C. Sotero, "Topological properties of restingstate fmri functional networks improve machine learning-based autism classification," Frontiers in neuroscience, vol. 12, p. 1018, 2019.

[9] O. Dekhil, M. Ali, R. Haweel, Y. Elnakib, M. Ghazal, H. Hajjdiab, L. Fraiwan, A. Shalaby, A. Soliman, A. Mahmoud et al., "A comprehensive framework for differentiating autism spectrum disorder from neurotypicals by fusing structural mri and resting state functional mri,' in Seminars in Pediatric Neurology. Elsevier, 2020, p. 100805

[10] B. G. Travers, N. Adluru, C. Ennis, D. P. Tromp, D. Destiche, S. Doran, E. D. Bigler, N. Lange, J. E. Lainhart, and A. L. Alexander, "Diffusion tensor imaging in autism spectrum disorder: a review," Autism Research, vol. 5, no. 5, pp. 289-313, 2012.

[11] J. Vicnesh, J. K. E. Wei, S. L. Oh, N. Arunkumar, E. W. Abdulhay, E. J. Ciaccio, U. R. Acharya et al., "Autism spectrum disorder diagnostic system using hos bispectrum with eeg signals," International Journal of Environmental Research and Public Health, vol. 17, no. 3, p. 971, 2020.

[12] D. Haputhanthri, G. Brihadiswaran, S. Gunathilaka, D. Meedeniya, S. Jayarathna, M. Jaime, and C. Harshaw, "Integration of facia thermography in eeg-based classification of asd," International Journal of Automation and Computing, vol. 17, pp. 1-18, 2020.

[13] J. Kang, T. Zhou, J. Han, and X. Li, "Eeg-based multi-feature fusion assessment for autism," Journal of Clinical Neuroscience, vol. 56, pp. 101-107, 2018.

[14] T. Sinha, M. V. Munot, and R. Sreemathy, "An efficient approach for detection of autism spectrum disorder using electroencephalography signal," IETE Journal of Research, pp. 1-9, 2019.

[15] M. Sadeghi, R. Khosrowabadi, F. Bakouie, H. Mahdavi, C. Eslahchi, and H. Pouretemad, "Screening of autism based on task-free fmri using graph theoretical approach," Psychiatry Research: Neuroimaging, vol. 263, pp. 48-56, 2017.

[16] A. Kazeminejad and R. C. Sotero, "Topological properties of restingstate fmri functional networks improve machine learning-based autism classification," Frontiers in neuroscience, vol. 12, p. 1018, 2019.

[17] J. C. Siero, D. Hermes, H. Hoogduin, P. R. Luijten, N. F. Ramsey, and N. Petridou, "Bold matches neuronal activity at the $\mathrm{mm}$ scale: A combined $7 \mathrm{t}$ fmri and ecog study in human sensorimotor cortex," Neuroimage, vol. 101, pp. 177-184, 2014.

[18] L. Xu, Q. Hua, J. Yu, and J. Li, "Classification of autism spectrum disorder based on sample entropy of spontaneous functional near infrared spectroscopy signal," Clinical Neurophysiology, 2020.

[19] R. G. Port, J. C. Edgar, M. Ku, L. Bloy, R. Murray, L. Blaskey, S. E. Levy, and T. P. Roberts, "Maturation of auditory neural processes in autism spectrum disorder-a longitudinal meg study," NeuroImage: Clinical, vol. 11, pp. 566-577, 2016.

[20] R. C. Deo, "Machine learning in medicine," Circulation, vol. 132, no. 20, pp. 1920-1930, 2015

[21] I. Goodfellow, Y. Bengio, and A. Courville, Deep learning. MIT press, 2016.
[22] J. Dolz, C. Desrosiers, L. Wang, J. Yuan, D. Shen, and I. B. Ayed, "Deep cnn ensembles and suggestive annotations for infant brain mri segmentation," Computerized Medical Imaging and Graphics, vol. 79, p. 101660,2020 .

[23] N. Ghassemi, A. Shoeibi, and M. Rouhani, "Deep neural network with generative adversarial networks pre-training for brain tumor classification based on mr images," Biomedical Signal Processing and Control, vol. 57, p. 101678, 2020.

[24] X. Li, N. C. Dvornek, X. Papademetris, J. Zhuang, L. H. Staib, P. Ventola, and J. S. Duncan, "2-channel convolutional 3d deep neural network (2cc3d) for fmri analysis: Asd classification and feature learning," in 2018 IEEE 15th International Symposium on Biomedical Imaging (ISBI 2018). IEEE, 2018, pp. 1252-1255.

[25] Q. Delannoy, C.-H. Pham, C. Cazorla, C. Tor-Díez, G. Dollé, H. Meunier, N. Bednarek, R. Fablet, N. Passat, and F. Rousseau, "Segsrgan: Super-resolution and segmentation using generative adversarial networks-application to neonatal brain mri," Computers in Biology and Medicine, p. 103755, 2020.

[26] R. Reed and R. J. MarksII, Neural smithing: supervised learning in feedforward artificial neural networks. Mit Press, 1999.

[27] G. E. Hinton, T. J. Sejnowski, T. A. Poggio et al., Unsupervised learning: foundations of neural computation. MIT press, 1999.

[28] M. Wiering and M. Van Otterlo, Reinforcement learning. Springer, 2012, vol. 12.

[29] J. Eldridge, A. E. Lane, M. Belkin, and S. Dennis, "Robust features for the automatic identification of autism spectrum disorder in children," Journal of neurodevelopmental disorders, vol. 6, no. 1, p. 12, 2014.

[30] S. Bhat, U. R. Acharya, H. Adeli, G. M. Bairy, and A. Adeli, "Autism: cause factors, early diagnosis and therapies," Reviews in the Neurosciences, vol. 25, no. 6, pp. 841-850, 2014.

[31] A. Dejman, A. Khadem, and A. Khorrami, "Exploring the disorders of brain effective connectivity network in asd: A case study using eeg, transfer entropy, and graph theory," in 2017 Iranian Conference on Electrical Engineering (ICEE). IEEE, 2017, pp. 8-13.

[32] N. Ghassemi, A. Shoeibi, M. Rouhani, and H. Hosseini-Nejad, "Epileptic seizures detection in eeg signals using tqwt and ensemble learning," in 2019 9th International Conference on Computer and Knowledge Engineering (ICCKE). IEEE, 2019, pp. 403-408.

[33] S. Bhat, U. R. Acharya, H. Adeli, G. M. Bairy, and A. Adeli, "Automated diagnosis of autism: in search of a mathematical marker," Reviews in the Neurosciences, vol. 25, no. 6, pp. 851-861, 2014.

[34] F. Thabtah, "Machine learning in autistic spectrum disorder behavioral research: A review and ways forward," Informatics for Health and Social Care, vol. 44, no. 3, pp. 278-297, 2019.

[35] D. Eman and A. W. Emanuel, "Machine learning classifiers for autism spectrum disorder: A review," in 2019 4th International Conference on Information Technology, Information Systems and Electrical Engineering (ICITISEE). IEEE, 2019, pp. 255-260.

[36] "Autism brain imaging data exchange i," http://fcon_1000.projects. nitrc.org/indi/abide/abide_I.html. 2016.

[37] "Abide preprocessed," http://preprocessed-connectomes-project.org/ abide/

[38] N. Payakachat, J. M. Tilford, and W. J. Ungar, "National database for autism research (ndar): big data opportunities for health services research and health technology assessment," Pharmacoeconomics, vol. 34, no. 2, pp. 127-138, 2016.

[39] M. Jenkinson, C. F. Beckmann, T. E. Behrens, M. W. Woolrich, and S. M. Smith, "Fsl," Neuroimage, vol. 62, no. 2, pp. 782-790, 2012.

[40] V. Popescu, M. Battaglini, W. Hoogstrate, S. C. Verfaillie, I. Sluimer, R. A. van Schijndel, B. W. van Dijk, K. S. Cover, D. L. Knol, M. Jenkinson et al., "Optimizing parameter choice for fsl-brain extraction tool (bet) on 3d t1 images in multiple sclerosis," Neuroimage, vol. 61, no. 4, pp. 1484-1494, 2012.

[41] B. Fischl, "Freesurfer," Neuroimage, vol. 62, no. 2, pp. 774-781, 2012.

[42] J. Ashburner, "Computational anatomy with the spm software," Magnetic resonance imaging, vol. 27, no. 8, pp. 1163-1174, 2009.

[43] H. A. Jaber, H. K. Aljobouri, İ. Çankaya, O. M. Koçak, and O. Algin, "Preparing fmri data for postprocessing: Conversion modalities, preprocessing pipeline, and parametric and nonparametric approaches," IEEE Access, vol. 7, pp. 122 864-122 877, 2019.

[44] M. Behroozi, M. R. Daliri, and H. Boyaci, "Statistical analysis methods for the fmri data," Basic and Clinical Neuroscience, vol. 2, no. 4, pp. 67-74, 2011.

[45] B.-y. Park, K. Byeon, and H. Park, "Funp (fusion of neuroimaging preprocessing) pipelines: a fully automated preprocessing software for functional magnetic resonance imaging," Frontiers in neuroinformatics, vol. 13 , p. $5,2019$. 
[46] J. A. Maldjian, P. J. Laurienti, R. A. Kraft, and J. H. Burdette, "An automated method for neuroanatomic and cytoarchitectonic atlas-based interrogation of fmri data sets," Neuroimage, vol. 19, no. 3, pp. 12331239, 2003.

[47] N. Tzourio-Mazoyer, B. Landeau, D. Papathanassiou, F. Crivello, O. Etard, N. Delcroix, B. Mazoyer, and M. Joliot, "Automated anatomical labeling of activations in spm using a macroscopic anatomical parcellation of the mni mri single-subject brain," Neuroimage, vol. 15 , no. 1, pp. 273-289, 2002.

[48] S. B. Eickhoff, K. E. Stephan, H. Mohlberg, C. Grefkes, G. R. Fink, K. Amunts, and K. Zilles, "A new spm toolbox for combining probabilistic cytoarchitectonic maps and functional imaging data," Neuroimage, vol. 25, no. 4, pp. 1325-1335, 2005.

[49] R. S. Desikan, F. Ségonne, B. Fischl, B. T. Quinn, B. C. Dickerson, D. Blacker, R. L. Buckner, A. M. Dale, R. P. Maguire, B. T. Hyman et al., "An automated labeling system for subdividing the human cerebral cortex on mri scans into gyral based regions of interest," Neuroimage, vol. 31, no. 3, pp. 968-980, 2006.

[50] J. Talairach, "Co-planar stereotaxic atlas of the human brain-3dimensional proportional system," An approach to cerebral imaging, 1988.

[51] N. U. Dosenbach, B. Nardos, A. L. Cohen, D. A. Fair, J. D. Power, J. A. Church, S. M. Nelson, G. S. Wig, A. C. Vogel, C. N. LessovSchlaggar et al., "Prediction of individual brain maturity using fmri," Science, vol. 329, no. 5997, pp. 1358-1361, 2010.

[52] R. C. Craddock, G. A. James, P. E. Holtzheimer III, X. P. Hu, and H. S Mayberg, "A whole brain fmri atlas generated via spatially constrained spectral clustering," Human brain mapping, vol. 33, no. 8, pp. 19141928, 2012.

[53] M. Kunda, S. Zhou, G. Gong, and H. Lu, "Improving multi-site autism classification based on site-dependence minimisation and second-order functional connectivity," bioRxiv, 2020.

[54] "Abide preprocessed - functional preprocessing," http: //preprocessed-connectomes-project.org/abide/Pipelines.html

[55] P. Bellec, C. Chu, F. Chouinard-Decorte, Y. Benhajali, D. S. Margulies, and R. C. Craddock, "The neuro bureau adhd-200 preprocessed repository," Neuroimage, vol. 144, pp. 275-286, 2017.

[56] C. Yan and Y. Zang, "Dparsf: a matlab toolbox for" pipeline" data analysis of resting-state fmri," Frontiers in systems neuroscience, vol. 4, p. 13, 2010.

[57] C. Craddock, S. Sikka, B. Cheung, R. Khanuja, S. S. Ghosh, C. Yan, Q. Li, D. Lurie, J. Vogelstein, R. Burns et al., "Towards automated analysis of connectomes: The configurable pipeline for the analysis of connectomes (c-pac)," Front Neuroinform, vol. 42, 2013.

[58] T. Xu, Z. Yang, L. Jiang, X.-X. Xing, and X.-N. Zuo, "A connectome computation system for discovery science of brain," Science Bulletin, vol. 60, no. 1, pp. 86-95, 2015.

[59] N. C. Dvornek, D. Yang, P. Ventola, and J. S. Duncan, "Learning generalizable recurrent neural networks from small task-fmri datasets," in International Conference on Medical Image Computing and ComputerAssisted Intervention. Springer, 2018, pp. 329-337.

[60] T. Eslami and F. Saeed, "Auto-asd-network: a technique based on deep learning and support vector machines for diagnosing autism spectrum disorder using fmri data," in Proceedings of the 10th ACM International Conference on Bioinformatics, Computational Biology and Health Informatics, 2019, pp. 646-651.

[61] Z. Sherkatghanad, M. Akhondzadeh, S. Salari, M. ZomorodiMoghadam, M. Abdar, U. R. Acharya, R. Khosrowabadi, and V. Salari, "Automated detection of autism spectrum disorder using a convolutional neural network," Frontiers in Neuroscience, vol. 13, 2019.

[62] M. A. Aghdam, A. Sharifi, and M. M. Pedram, "Diagnosis of autism spectrum disorders in young children based on resting-state functional magnetic resonance imaging data using convolutional neural networks," Journal of digital imaging, vol. 32, no. 6, pp. 899-918, 2019.

[63] O. Dekhil, H. Hajjdiab, B. Ayinde, A. Shalaby, A. Switala, D. Sosnin, A. Elshamekh, M. Ghazal, R. Keynton, G. Barnes et al., "Using resting state functional mri to build a personalized autism diagnosis system," in 2018 IEEE 15th International Symposium on Biomedical Imaging (ISBI 2018). IEEE, 2018, pp. 1381-1385.

[64] F. Chollet, Deep Learning mit Python und Keras: Das PraxisHandbuch vom Entwickler der Keras-Bibliothek. MITP-Verlags GmbH \& Co. KG, 2018.

[65] L. Zou, J. Zheng, C. Miao, M. J. Mckeown, and Z. J. Wang, "3d cnn based automatic diagnosis of attention deficit hyperactivity disorder using functional and structural mri," IEEE Access, vol. 5, pp. 23626 $23636,2017$.
[66] A. Radford, L. Metz, and S. Chintala, "Unsupervised representation learning with deep convolutional generative adversarial networks," arXiv preprint arXiv:1511.06434, 2015.

[67] C. Doersch, "Tutorial on variational autoencoders," arXiv preprint arXiv:1606.05908, 2016.

[68] Z. Che, S. Purushotham, K. Cho, D. Sontag, and Y. Liu, "Recurrent neural networks for multivariate time series with missing values," Scientific reports, vol. 8, no. 1, pp. 1-12, 2018.

[69] J. Wang, Y. Yang, J. Mao, Z. Huang, C. Huang, and W. Xu, "Cnn-rnn: A unified framework for multi-label image classification," in Proceedings of the IEEE conference on computer vision and pattern recognition, 2016, pp. 2285-2294.

[70] Y. Fan, X. Lu, D. Li, and Y. Liu, "Video-based emotion recognition using cnn-rnn and c3d hybrid networks," in Proceedings of the 18th ACM International Conference on Multimodal Interaction, 2016, pp. 445-450.

[71] M. Chen, X. Shi, Y. Zhang, D. Wu, and M. Guizani, "Deep features learning for medical image analysis with convolutional autoencoder neural network," IEEE Transactions on Big Data, 2017.

[72] M. I. U. Haque and D. Valles, "Facial expression recognition using denn and development of an ios app for children with asd to enhance communication abilities," in 2019 IEEE 10th Annual Ubiquitous Computing, Electronics \& Mobile Communication Conference (UEMCON). IEEE, 2019, pp. 0476-0482.

[73] M. I. U. Haque and D. Valles, "Facial expression recognition from different angles using denn for children with asd to identify emotions," in 2018 International Conference on Computational Science and Computational Intelligence (CSCI). IEEE, 2018, pp. 446-449.

[74] N. M. Rad, S. M. Kia, C. Zarbo, T. van Laarhoven, G. Jurman, P. Venuti, E. Marchiori, and C. Furlanello, "Deep learning for automatic stereotypical motor movement detection using wearable sensors in autism spectrum disorders," Signal Processing, vol. 144, pp. 180-191, 2018.

[75] C. Wu, S. Liaqat, S.-c. Cheung, C.-N. Chuah, and S. Ozonoff, "Predicting autism diagnosis using image with fixations and synthetic saccade patterns," in 2019 IEEE International Conference on Multimedia \& Expo Workshops (ICMEW). IEEE, 2019, pp. 647-650.

[76] M. Elbattah, R. Carette, G. Dequen, J.-L. Guérin, and F. Cilia, "Learning clusters in autism spectrum disorder: Image-based clustering of eye-tracking scanpaths with deep autoencoder," in 201941 st Annual International Conference of the IEEE Engineering in Medicine and Biology Society (EMBC). IEEE, 2019, pp. 1417-1420.

[77] X. Li, N. C. Dvornek, Y. Zhou, J. Zhuang, P. Ventola, and J. S. Duncan, "Efficient interpretation of deep learning models using graph structure and cooperative game theory: Application to asd biomarker discovery," in International Conference on Information Processing in Medical Imaging. Springer, 2019, pp. 718-730.

[78] Y. Zhao, Q. Dong, S. Zhang, W. Zhang, H. Chen, X. Jiang, L. Guo, $\mathrm{X}$. Hu, J. Han, and T. Liu, "Automatic recognition of fmri-derived functional networks using 3-d convolutional neural networks," IEEE Transactions on Biomedical Engineering, vol. 65, no. 9, pp. 19751984, 2017.

[79] M. Leming, J. M. Górriz, and J. Suckling, "Ensemble deep learning on large, mixed-site fmri datasets in autism and other tasks," arXiv preprint arXiv:2002.07874, 2020.

[80] X. Li, N. C. Dvornek, J. Zhuang, P. Ventola, and J. S. Duncan, "Brain biomarker interpretation in asd using deep learning and fmri," in International Conference on Medical Image Computing and ComputerAssisted Intervention. Springer, 2018, pp. 206-214.

[81] M. Khosla, K. Jamison, A. Kuceyeski, and M. R. Sabuncu, "3d convolutional neural networks for classification of functional connectomes," in Deep Learning in Medical Image Analysis and Multimodal Learning for Clinical Decision Support. Springer, 2018, pp. 137-145.

[82] F. Saeed, T. Eslami, V. Mirjalili, A. Fong, and A. Laird, "Asd-diagnet: A hybrid learning approach for detection of autism spectrum disorder using fmri data," Frontiers in Neuroinformatics, vol. 13, p. 70, 2019.

[83] R. Anirudh and J. J. Thiagarajan, "Bootstrapping graph convolutional neural networks for autism spectrum disorder classification," in ICASSP 2019-2019 IEEE International Conference on Acoustics, Speech and Signal Processing (ICASSP). IEEE, 2019, pp. 3197-3201.

[84] C. J. Brown, J. Kawahara, and G. Hamarneh, "Connectome priors in deep neural networks to predict autism," in 2018 IEEE 15th International Symposium on Biomedical Imaging (ISBI 2018). IEEE, 2018, pp. 110-113.

[85] D. Liao and H. Lu, "Classify autism and control based on deep learning and community structure on resting-state fmri," in 2018 Tenth Interna- 
tional Conference on Advanced Computational Intelligence (ICACI). IEEE, 2018, pp. 289-294.

[86] X. Yang, S. Sarraf, and N. Zhang, "Deep learning-based framework for autism functional mri image classification," Journal of the Arkansas Academy of Science, vol. 72, no. 1, pp. 47-52, 2018

[87] X. Guo, K. C. Dominick, A. A. Minai, H. Li, C. A. Erickson, and L. J. Lu, "Diagnosing autism spectrum disorder from brain restingstate functional connectivity patterns using a deep neural network with a novel feature selection method," Frontiers in neuroscience, vol. 11, p. 460, 2017.

[88] M. Khosla, K. Jamison, A. Kuceyeski, and M. R. Sabuncu, "Ensemble learning with $3 \mathrm{~d}$ convolutional neural networks for functional connectome-based prediction," Neuroimage, vol. 199, pp. 651-662, 2019

[89] H. Choi, "Functional connectivity patterns of autism spectrum disorder identified by deep feature learning," arXiv preprint arXiv:1707.07932, 2017.

[90] N. C. Dvornek, P. Ventola, K. A. Pelphrey, and J. S. Duncan, "Identifying autism from resting-state fmri using long short-term memory networks," in International Workshop on Machine Learning in Medical Imaging. Springer, 2017, pp. 362-370.

[91] H. Lu, S. Liu, H. Wei, and J. Tu, "Multi-kernel fuzzy clustering based on auto-encoder for fmri functional network," Expert Systems with Applications, p. 113513, 2020.

[92] Z. Xiao, C. Wang, N. Jia, and J. Wu, "Sae-based classification of school-aged children with autism spectrum disorders using functional magnetic resonance imaging," Multimedia Tools and Applications, vol. 77 , no. 17 , pp. $22809-22820,2018$.

[93] N. C. Dvornek, X. Li, J. Zhuang, and J. S. Duncan, "Jointly discriminative and generative recurrent neural networks for learning from fmri," in International Workshop on Machine Learning in Medical Imaging. Springer, 2019, pp. 382-390.

[94] K. Niu, J. Guo, Y. Pan, X. Gao, X. Peng, N. Li, and H. Li, "Multichannel deep attention neural networks for the classification of autism spectrum disorder using neuroimaging and personal characteristic data," Complexity, vol. 2020, 2020.

[95] H. Li, N. A. Parikh, and L. He, "A novel transfer learning approach to enhance deep neural network classification of brain functional connectomes," Frontiers in neuroscience, vol. 12, p. 491, 2018.

[96] A. El Gazzar, L. Cerliani, G. van Wingen, and R. M. Thomas, "Simple 1-d convolutional networks for resting-state fmri based classification in autism," in 2019 International Joint Conference on Neural Networks (IJCNN). IEEE, 2019, pp. 1-6.

[97] M. R. Ahmed, Y. Zhang, Y. Liu, and H. Liao, "Single volume image generator and deep learning-based asd classification," IEEE Journal of Biomedical and Health Informatics, 2020.

[98] Y. Zhao, H. Dai, W. Zhang, F. Ge, and T. Liu, "Two-stage spatial temporal deep learning framework for functional brain network modeling," in 2019 IEEE 16th International Symposium on Biomedical Imaging (ISBI 2019). IEEE, 2019, pp. 1576-1580.

[99] B. Pugazhenthi, G. Senapathy, and M. Pavithra, "Identification of autism in mr brain images using deep learning networks," in 2019 International Conference on Smart Structures and Systems (ICSSS). IEEE, 2019, pp. 1-7.

[100] T. Eslami, J. S. Raiker, and F. Saeed, "Explainable and scalable machine-learning algorithms for detection of autism spectrum disorder using fmri data," arXiv preprint arXiv:2003.01541, 2020.

[101] K. Sairam, J. Naren, G. Vithya, and S. Srivathsan, "Computer aided system for autism spectrum disorder using deep learning methods," International Journal of Psychosocial Rehabilitation, vol. 23, no. 01, 2019.

[102] J. Dolz, C. Desrosiers, and I. B. Ayed, "3d fully convolutional networks for subcortical segmentation in mri: A large-scale study," NeuroImage, vol. 170, pp. 456-470, 2018.

[103] C. Wang, Z. Xiao, B. Wang, and J. Wu, "Identification of autism based on svm-rfe and stacked sparse auto-encoder," IEEE Access, vol. 7, pp. $118030-118036,2019$.

[104] Y. Zhao, F. Ge, S. Zhang, and T. Liu, "3d deep convolutional neural network revealed the value of brain network overlap in differentiating autism spectrum disorder from healthy controls," in International Conference on Medical Image Computing and Computer-Assisted Intervention. Springer, 2018, pp. 172-180.

[105] S. Parisot, S. I. Ktena, E. Ferrante, M. Lee, R. Guerrero, B. Glocker and D. Rueckert, "Disease prediction using graph convolutional networks: Application to autism spectrum disorder and alzheimer's disease," Medical image analysis, vol. 48, pp. 117-130, 2018.
[106] N. C. Dvornek, P. Ventola, and J. S. Duncan, "Combining phenotypic and resting-state fmri data for autism classification with recurrent neural networks," in 2018 IEEE 15th International Symposium on Biomedical Imaging (ISBI 2018). IEEE, 2018, pp. 725-728.

[107] A. S. Heinsfeld, A. R. Franco, R. C. Craddock, A. Buchweitz, and F. Meneguzzi, "Identification of autism spectrum disorder using deep learning and the abide dataset," NeuroImage: Clinical, vol. 17, pp. 1623, 2018.

[108] X. Li, J. Hect, M. Thomason, and D. Zhu, "Interpreting age effects of human fetal brain from spontaneous fmri using deep $3 \mathrm{~d}$ convolutional neural networks," in 2020 IEEE 17th International Symposium on Biomedical Imaging (ISBI). IEEE, 2020, pp. 1424-1427.

[109] M. A. Aghdam, A. Sharifi, and M. M. Pedram, "Combination of rsfmri and smri data to discriminate autism spectrum disorders in young children using deep belief network," Journal of digital imaging, vol. 31, no. 6, pp. 895-903, 2018

[110] C. Mellema, A. Treacher, K. Nguyen, and A. Montillo, "Multiple deep learning architectures achieve superior performance diagnosing autism spectrum disorder using features previously extracted from structural and functional mri," in 2019 IEEE 16th International Symposium on Biomedical Imaging (ISBI 2019). IEEE, 2019, pp. 1891-1895.

[111] M. Rakić, M. Cabezas, K. Kushibar, A. Oliver, and X. Lladó, "Improving the detection of autism spectrum disorder by combining structural and functional mri information," NeuroImage: Clinical, vol. 25, p. 102181, 2020.

[112] G. Li, M. Liu, Q. Sun, D. Shen, and L. Wang, "Early diagnosis of autism disease by multi-channel cnns," in International Workshop on Machine Learning in Medical Imaging. Springer, 2018, pp. 303-309.

[113] G. Li, M.-H. Chen, G. Li, D. Wu, Q. Sun, D. Shen, and L. Wang, "A preliminary volumetric mri study of amygdala and hippocampal subfields in autism during infancy," in 2019 IEEE 16th International Symposium on Biomedical Imaging (ISBI 2019). IEEE, 2019, pp. 1052-1056.

[114] M. Ismail, G. Barnes, M. Nitzken, A. Switala, A. Shalaby, E. HosseiniAsl, M. Casanova, R. Keynton, A. Khalil, and A. El-Baz, "A new deep-learning approach for early detection of shape variations in autism using structural mri," in 2017 IEEE International Conference on Image Processing (ICIP). IEEE, 2017, pp. 1057-1061.

[115] Y. Kong, J. Gao, Y. Xu, Y. Pan, J. Wang, and J. Liu, "Classification of autism spectrum disorder by combining brain connectivity and deep neural network classifier," Neurocomputing, vol. 324, pp. 63-68, 2019.

[116] W. H. Pinaya, A. Mechelli, and J. R. Sato, "Using deep autoencoders to identify abnormal brain structural patterns in neuropsychiatric disorders: A large-scale multi-sample study," Human brain mapping, vol. 40, no. 3, pp. 944-954, 2019.

[117] S. J. Sujit, I. Coronado, A. Kamali, P. A. Narayana, and R. E. Gabr, "Automated image quality evaluation of structural brain mri using an ensemble of deep learning networks," Journal of Magnetic Resonance Imaging, vol. 50, no. 4, pp. 1260-1267, 2019.

[118] L. Henschel, S. Conjeti, S. Estrada, K. Diers, B. Fischl, and M. Reuter, "Fastsurfer-a fast and accurate deep learning based neuroimaging pipeline," NeuroImage, p. 117012, 2020.

[119] H. Shahamat and M. S. Abadeh, "Brain mri analysis using a deep learning based volutionary approach," Neural Networks, 2020.

[120] J. E. Iglesias, G. Lerma-Usabiaga, L. C. Garcia-Peraza-Herrera, S. Martinez, and P. M. Paz-Alonso, "Retrospective head motion estimation in structural brain mri with $3 \mathrm{~d}$ cnns," in International Conference on Medical Image Computing and Computer-Assisted Intervention. Springer, 2017, pp. 314-322.

[121] K. Byeon, J. Kwon, J. Hong, and H. Park, "Artificial neural network inspired by neuroimaging connectivity: Application in autism spectrum disorder," in 2020 IEEE International Conference on Big Data and Smart Computing (BigComp). IEEE, 2020, pp. 575-578.

[122] L. Xu, Y. Liu, J. Yu, X. Li, X. Yu, H. Cheng, and J. Li, "Characterizing autism spectrum disorder by deep learning spontaneous brain activity from functional near-infrared spectroscopy," Journal of Neuroscience Methods, vol. 331, p. 108538, 2020.

[123] L. Xu, X. Geng, X. He, J. Li, and J. Yu, "Prediction in autism by deep learning short-time spontaneous hemodynamic fluctuations," Frontiers in Neuroscience, vol. 13, 2019.

[124] B. Thyreau and Y. Taki, "Learning a cortical parcellation of the brain robust to the mri segmentation with convolutional neural networks," Medical Image Analysis, vol. 61, p. 101639, 2020.

[125] R. M. Thomas, S. Gallo, L. Cerliani, P. Zhutovsky, A. El-Gazzar, and G. van Wingen, "Classifying autism spectrum disorder using the temporal statistics of resting-state functional mri data with $3 \mathrm{~d}$ 
convolutional neural networks," Frontiers in Psychiatry, vol. 11, p. 440, 2020.

[126] A. El-Gazzar, M. Quaak, L. Cerliani, P. Bloem, G. van Wingen, and R. M. Thomas, "A hybrid 3dcnn and 3dc-lstm based model for $4 \mathrm{~d}$ spatio-temporal fmri data: An abide autism classification study," in OR 2.0 Context-Aware Operating Theaters and Machine Learning in Clinical Neuroimaging. Springer, 2019, pp. 95-102.

[127] S. Mostafa, W. Yin, and F.-X. Wu, "Autoencoder based methods for diagnosis of autism spectrum disorder," in International Conference on Computational Advances in Bio and Medical Sciences. Springer, 2019, pp. 39-51.

[128] Z. Jiao, H. Li, and Y. Fan, "Improving diagnosis of autism spectrum disorder and disentangling its heterogeneous functional connectivity patterns using capsule networks," in 2020 IEEE 17th International Symposium on Biomedical Imaging (ISBI). IEEE, 2020, pp. 13311334.

[129] J. Xie, L. Wang, P. Webster, Y. Yao, J. Sun, S. Wang, and H. Zhou, "A two-stream end-to-end deep learning network for recognizing atypical visual attention in autism spectrum disorder," arXiv preprint arXiv:1911.11393, 2019.

[130] S. Sadiq, M. Castellanos, J. Moffitt, M.-L. Shyu, L. Perry, and D. Messinger, "Deep learning based multimedia data mining for autism spectrum disorder (asd) diagnosis," in 2019 International Conference on Data Mining Workshops (ICDMW). IEEE, 2019, pp. 847-854.

[131] Y. Tao and M.-L. Shyu, "Sp-asdnet: Cnn-lstm based asd classification model using observer scanpaths," in 2019 IEEE International Conference on Multimedia \& Expo Workshops (ICMEW). IEEE, 2019, pp. 641-646.

[132] J. R. H. Lee and A. Wong, "Timeconvnets: A deep time windowed convolution neural network design for real-time video facial expression recognition," in 2020 17th Conference on Computer and Robot Vision (CRV). IEEE, 2020, pp. 9-16.

[133] A. Vijayan, S. Janmasree, C. Keerthana, and L. B. Syla, "A framework for intelligent learning assistant platform based on cognitive computing for children with autism spectrum disorder," in 2018 International CET Conference on Control, Communication, and Computing (IC4). IEEE, 2018, pp. 361-365.

[134] A. Di Nuovo, D. Conti, G. Trubia, S. Buono, and S. Di Nuovo, "Deep learning systems for estimating visual attention in robot-assisted therapy of children with autism and intellectual disability," Robotics, vol. 7, no. 2, p. 25, 2018.

[135] N. M. Rad, S. M. Kia, C. Zarbo, T. van Laarhoven, G. Jurman, P. Venuti, E. Marchiori, and C. Furlanello, "Deep learning for automatic stereotypical motor movement detection using wearable sensors in autism spectrum disorders," Signal Processing, vol. 144, pp. 180-191, 2018.

[136] S. Jaiswal, M. F. Valstar, A. Gillott, and D. Daley, "Automatic detection of adhd and asd from expressive behaviour in rgbd data," in 2017 12th IEEE International Conference on Automatic Face \& Gesture Recognition (FG 2017). IEEE, 2017, pp. 762-769.

[137] Y.-S. Liu, C.-P. Chen, S. S.-F. Gau, and C.-C. Lee, "Learning lexical coherence representation using lstm forget gate for children with autism spectrum disorder during story-telling," in 2018 IEEE International Conference on Acoustics, Speech and Signal Processing (ICASSP). IEEE, 2018, pp. 6029-6033.

[138] J. Li, Y. Zhong, J. Han, G. Ouyang, X. Li, and H. Liu, "Classifying asd children with 1stm based on raw videos," Neurocomputing, 2019.

[139] W. Wei, Z. Liu, L. Huang, A. Nebout, and O. Le Meur, "Saliency prediction via multi-level features and deep supervision for children with autism spectrum disorder," in 2019 IEEE International Conference on Multimedia \& Expo Workshops (ICMEW). IEEE, 2019, pp. 621624.

[140] S. Raj and S. Masood, "Analysis and detection of autism spectrum disorder using machine learning techniques," Procedia Computer Science, vol. 167, pp. 994-1004, 2020.

[141] O. Rudovic, Y. Utsumi, J. Lee, J. Hernandez, E. C. Ferrer, B. Schuller, and R. W. Picard, "Culturenet: A deep learning approach for engagement intensity estimation from face images of children with autism," in 2018 IEEE/RSJ International Conference on Intelligent Robots and Systems (IROS). IEEE, 2018, pp. 339-346.

[142] A. Cook, B. Mandal, D. Berry, and M. Johnson, "Towards automatic screening of typical and atypical behaviors in children with autism," in 2019 IEEE International Conference on Data Science and Advanced Analytics (DSAA). IEEE, 2019, pp. 504-510.

[143] H. Javed and C. H. Park, "Behavior-based risk detection of autism spectrum disorder through child-robot interaction," in Companion of the 2020 ACM/IEEE International Conference on Human-Robot Interaction, 2020, pp. 275-277.

[144] K. Sun, L. Li, L. Li, N. He, and J. Zhu, "Spatial attentional bilinear 3d convolutional network for video-based autism spectrum disorder detection," in ICASSP 2020-2020 IEEE International Conference on Acoustics, Speech and Signal Processing (ICASSP). IEEE, 2020, pp. 3387-3391.

[145] H. Wang, L. Li, L. Chi, and Z. Zhao, "Autism screening using deep embedding representation," in International Conference on Computational Science. Springer, 2019, pp. 160-173.

[146] M. F. Misman, A. A. Samah, F. A. Ezudin, H. A. Majid, Z. A. Shah, H. Hashim, and M. F. Harun, "Classification of adults with autism spectrum disorder using deep neural network," in 2019 1st International Conference on Artificial Intelligence and Data Sciences (AiDAS). IEEE, 2019, pp. 29-34. 